TITLE:

\title{
Vapor flows in the continuum limit in the presence of a small amount of noncondensable gas
}

\author{
$\operatorname{AUTHOR}(\mathrm{S})$ :
}

Taguchi, Satoshi; Aoki, Kazuo; Takata, Shigeru

\section{CITATION:}

Taguchi, Satoshi ...[et al]. Vapor flows in the continuum limit in the presence of a small amount of noncondensable gas. PHYSICS OF FLUIDS 2004, 16(11): 4105-4120

\section{ISSUE DATE:}

2004-11

URL:

http://hdl.handle.net/2433/39785

\section{RIGHT:}

Copyright 2004 American Institute of Physics. This article may be downloaded for personal use only. Any other use requires prior permission of the author and the American Institute of Physics. 


\title{
Vapor flows in the continuum limit in the presence of a small amount of noncondensable gas
}

\author{
Satoshi Taguchi, Kazuo Aoki, ${ }^{a)}$ and Shigeru Takata \\ Department of Aeronautics and Astronautics and Advanced Research Institute of Fluid Science \\ and Engineering, Graduate School of Engineering, Kyoto University, Kyoto 606-8501, Japan
}

(Received 11 May 2004; accepted 30 July 2004; published online 6 October 2004)

\begin{abstract}
Steady flows of a vapor around its condensed phase of arbitrary shape, on the surface of which evaporation and condensation of the vapor may take place, are considered in the presence of a small amount of a noncondensable gas. By a systematic asymptotic analysis of the Boltzmann system, the present authors have derived the fluid-dynamic system describing such flows in the continuum limit in the case where the amount of the noncondensable gas is infinitesimal compared with that of the vapor [K. Aoki, S. Takata, and S. Taguchi, Eur. J. Mech. B/Fluids 22, 51 (2003)]. In the present study, the fluid-dynamic system is applied to some physical problems, and it is demonstrated with concrete examples that such a tiny amount of the noncondensable gas causes dramatic changes of the overall vapor flows. () 2004 American Institute of Physics. [DOI: 10.1063/1.1795271]
\end{abstract}

\section{INTRODUCTION}

In flows of a vapor with evaporation or condensation taking place on the boundary, the vapor is not in a local equilibrium state near the boundary even in the continuum limit, i.e., the limit in which the Knudsen number, defined by the ratio of the mean free path of the vapor molecules to the characteristic length of the system, goes to zero. Therefore, an approach based on kinetic theory is required to describe such flows. In fact, this type of flow has been one of the important subjects in kinetic theory of gases for more than three decades. ${ }^{1-6}$

Concerning the continuum limit (and its neighborhood), the fluid-dynamic systems that describe steady flows of the vapor have been established by means of systematic asymptotic analyses of the Boltzmann system. ${ }^{5,7-10}$ Some of these fluid-dynamic systems have been extended to the vapor flows in the presence of another noncondensable gas that neither evaporates nor condenses on the boundary. ${ }^{11,12}$ However, some interesting and important phenomena peculiar to the continuum limit for the mixture of the vapor and the noncondensable gas were clarified only recently. ${ }^{13-18}$

The phenomenon that is investigated in Ref. 18 is the effect of a tiny amount of the noncondensable gas on the overall vapor flows. For the sake of simplicity we restrict ourselves to the case of a closed domain, and we suppose that steady evaporation and condensation are taking place on the boundary. Let $n_{r}$ be the reference molecular number density of the vapor, $n_{a v}^{B}$ the average molecular number density of the noncondensable gas over the domain, and $\mathrm{Kn}$ the Knudsen number defined by the mean free path of the vapor molecules in a reference equilibrium state divided by the characteristic length of the domain. We assume that the Knudsen number Kn is small and that the average concentration of the noncondensable gas is as small as the Knudsen number. The latter condition is equivalent to $n_{a v}^{B} / n_{r}=O(\mathrm{Kn})$.

a)Electronic mail: aoki@aero.mbox.media.kyoto-u.ac.jp
The fluid-dynamic equations, which are the set of compressible Euler equations, and their boundary conditions for the continuum limit under this condition were derived in Ref. 18 with the help of a systematic asymptotic analysis of the Boltzmann system for small $\mathrm{Kn}$ and a complementary numerical analysis performed in Refs. 19 and 20. In this limit, the average concentration becomes infinitesimal $\left(n_{a v}^{B} / n_{r} \rightarrow 0\right)$. However, it was shown that the noncondensable gas of an infinitesimal average concentration accumulates in the Knudsen layer with an infinitesimal thickness on the condensing boundary and has a significant effect on the overall vapor flow through the fluid-dynamic boundary condition on the condensing boundary. An example that demonstrates a dramatic change of the streamlines of the vapor flow caused by the presence of the noncondensable gas with an infinitesimal average concentration is also given in Ref. 18.

High-speed flows of a single-component vapor that undergo strong evaporation and condensation play an important role in various applications such as heat pipes and laser ablation. In practical situations, however, the vapor flows may contain tiny impurities. The fluid-dynamic system established in Ref. 18 reveals that such impurities have a crucial effect on the vapor flows. Moreover, it enables quantitative assessment of the effect of the impurities. Therefore, the results given in Ref. 18 are not only of theoretical interest but also of practical importance.

In the present paper, we will give some more applications of the fluid-dynamic system derived in Ref. 18 and demonstrate the effect of the trace of the noncondensable gas on the global vapor flow. We first summarize the fluiddynamic system (Sec. II) and then investigate three concrete problems numerically (Sec. III).

\section{BASIC EQUATIONS}

In this section, we first describe the physical situation that we consider and then summarize the fluid-dynamic equations and their boundary conditions obtained in Ref. 18. 


\section{A. Physical situation}

Let us consider a vapor and its condensed phase of arbitrarily smooth shape, on the surface of which evaporation or condensation of the vapor is taking place. For the sake of simplicity, we restrict ourselves to the case of a closed domain (see Ref. 18 for the case of an infinite domain). A small amount of the noncondensable gas is contained in the system. In the following, we assign the label $A$ to the vapor (it will also be called $A$ component) and $B$ to the noncondensable gas (it will also be called $B$ component).

We first introduce the reference quantities: $L$ is the reference length of the system, $n_{r}$ is the reference number density of the vapor molecules, $T_{r}$ is the reference temperature, $p_{r}$ is the reference pressure of the vapor defined by $p_{r}$ $=k n_{r} T_{r}$ ( $k$ is the Boltzmann constant), $m^{A}$ is the mass of a molecule of the vapor, $l_{r}$ is the mean free path of the vapor molecules in the equilibrium state at rest with temperature $T_{r}$ and number density $n_{r}$, and $\mathrm{Kn}=l_{r} / L$ is the Knudsen number with respect to the vapor. Further, we denote by $n_{a v}^{B}$ the average number density of the noncondensable gas contained in the system.

We consider steady flows of the vapor in the following situation: (i) The Knudsen number $\mathrm{Kn}$ is small. (ii) The amount of the noncondensable gas contained in the system is small; to be more specific, the amount is such that the average concentration of the noncondensable gas is of the order of the Knudsen number Kn. The latter condition is equivalent to setting

$$
\frac{n_{a v}^{B}}{n_{r}}=O(\mathrm{Kn})
$$

We will focus on the continuum limit where $\mathrm{Kn} \rightarrow 0$, or equivalently, the zeroth-order quantities in Kn. It should be noted that in the situation of Eq. (1), the average concentration of the noncondensable gas becomes infinitesimal in the continuum limit. Nevertheless, as we will see, its trace has an important effect on the overall flow of the vapor.

\section{B. Fluid-dynamic equations}

In Ref. 18, the asymptotic behavior of the vapor and the noncondensable gas was investigated in the situation described in Sec. II A. More specifically, a systematic asymptotic analysis of the Boltzmann equation for small Knudsen numbers has been carried out under the assumption that the interaction of the vapor molecules with the boundary is described by the conventional condition for evaporation and condensation (the so-called complete condensation condition), and that of the noncondensable-gas molecules with the boundary by the diffuse reflection condition. The result is summarized here and in Sec. II C.

Let $X_{i}$ denote the space rectangular coordinate system, $n^{\alpha}, \rho^{\alpha}$, and $p^{\alpha}$ the number density, the mass density, and the pressure of the $\alpha$ component $(\alpha=A, B)$, respectively, and $v_{i}$ and $T$ the flow velocity and the temperature of the vapor, respectively. We introduce their dimensionless counterparts by the following relations:

$$
\begin{aligned}
& x_{i}=X_{i} / L, \quad \hat{n}^{\alpha}=n^{\alpha} / n_{r}, \\
& \hat{\rho}^{\alpha}=\rho^{\alpha} / m^{A} n_{r}, \quad \hat{p}^{\alpha}=p^{\alpha} / p_{r}, \\
& \hat{v}_{i}=v_{i} /\left(2 k T_{r} / m^{A}\right)^{1 / 2}, \quad \hat{T}=T / T_{r} .
\end{aligned}
$$

According to Ref. 18, the macroscopic quantities $h$ ( $h$ $=\hat{n}^{\alpha}, \hat{\rho}^{\alpha}, \hat{p}^{\alpha}, \hat{v}_{i}$, or $\left.\hat{T}\right)$ are expressed in the following form:

$$
h=h_{H}+h_{K},
$$

where $h_{H}$ is the moderately varying overall solution whose length scale of variation is the reference length of the system [i.e., $\left.\partial h_{H} / \partial x_{i}=O(1)\right]$ and $h_{K}$ is a correction term to $h_{H}$ in a thin layer (the Knudsen layer) with thickness of the order of the mean free path (or of the order of $\mathrm{Kn}$ in the dimensionless $x_{i}$ space) adjacent to the boundary. The $h_{H}$ is the socalled Hilbert solution, and we call $h_{K}$ the Knudsen-layer correction. We consider the situation in which there is no closed streamlines of the vapor flow or in which closed streamlines of the vapor flow, if any, do not carry any noncondensable gas. Then, as discussed in Ref. 18, we can assume, consistently with Eq. (1), that the noncondensable gas is absent in the overall flow field, that is,

$$
\hat{n}_{H}^{B}=\hat{\rho}_{H}^{B}=\hat{p}_{H}^{B}=0,
$$

and that the noncondensable gas is also absent in the Knudsen layer on the evaporating boundary (this fact can be shown rigorously for the Maxwell molecules and for most of the model equations ${ }^{18}$ ). Consequently, the noncondensable gas can be present only in the Knudsen layer on the condensing boundary.

The macroscopic quantities $h_{H}$ in the continuum limit $\mathrm{Kn} \rightarrow 0$ (or, equivalently, the zeroth-order terms of their Knexpansions) are described by the Euler set of equations for an ideal gas, which is written in the following dimensionless form: ${ }^{18}$

$$
\begin{aligned}
& \frac{\partial \hat{\rho}^{A} \hat{v}_{j}}{\partial x_{j}}=0, \\
& \hat{\rho}^{A} \hat{v}_{j} \frac{\partial \hat{v}_{i}}{\partial x_{j}}+\frac{1}{2} \frac{\partial \hat{p}^{A}}{\partial x_{i}}=0, \\
& \hat{v}_{j} \frac{\partial}{\partial x_{j}}\left(\frac{5}{2} \hat{T}+\hat{v}_{k}^{2}\right)=0, \\
& \hat{p}^{A}=\hat{\rho}^{A} \hat{T},
\end{aligned}
$$

where $\hat{\rho}^{A}=\hat{n}^{A}$. Here and in what follows, we omit the subscript $H$ for the Hilbert solution because no confusion is expected.

\section{Boundary conditions}

The Knudsen-layer correction $h_{K}$ [see Eq. (3)] is obtained by solving a half-space boundary-value problem of 
the Boltzmann equation. More specifically, the Knudsenlayer correction for the evaporating surface corresponds to the solution for the steady flow of a pure vapor evaporating from a plane condensed phase, ${ }^{21-24}$ and that for the condensing surface corresponds to the solution for the steady flow of a vapor condensing onto a plane condensed phase in the presence of the noncondensable gas. ${ }^{19,20,25,26}$ The boundary conditions for the Euler equations (5a)-(5d) are obtained together with the solution for the Knudsen-layer corrections. We will summarize, in the following, the boundary conditions thus obtained (see Ref. 18 for the details of the derivation).

Let $L x_{w i}\left(\chi_{1}, \chi_{2}\right)$ denote the boundary, where $\chi_{1}$ and $\chi_{2}$ are the (dimensionless) coordinates on the boundary orthogonal to each other, $T_{r} \hat{T}_{w}$ the temperature of the boundary, $\left(2 k T_{r} / m^{A}\right)^{1 / 2} \hat{v}_{w i}$ its velocity, and $p_{r} \hat{p}_{w}^{A}$ the saturation pressure of the vapor at temperature $T_{r} \hat{T}_{w}$. Further, we denote by $n_{i}$ the unit normal vector to the boundary pointing to the gas region and by $t_{i}^{(1)}$ and $t_{i}^{(2)}$ the unit tangential vectors to the boundary in the direction of increasing $\chi_{1}$ and $\chi_{2}$, which are taken in such a way that $t_{i}^{(1)}, t_{i}^{(2)}$, and $n_{i}$ form a right-hand system. Since we consider steady flows, $\hat{v}_{w i} n_{i}=0$ holds.

First, we introduce the following local Mach numbers based on the normal and tangential flow velocities on the boundary relative to the velocity of the boundary:

$$
\begin{aligned}
& M_{n}=\sqrt{6 / 5}(\hat{T})_{b}^{-1 / 2}\left|\left(\hat{v}_{j}\right)_{b} n_{j}\right|, \\
& M_{t}=\sqrt{6 / 5}(\hat{T})_{b}^{-1 / 2}\left|\left(\hat{v}_{i}\right)_{b}-\hat{v}_{w i}-\left(\hat{v}_{j}\right)_{b} n_{j} n_{i}\right|,
\end{aligned}
$$

where $(\cdot)_{b}$ indicates that the quantity in the parentheses is evaluated on the boundary. Then, the boundary conditions for the Euler equations in the continuum limit are expressed as follows.

\section{Boundary condition on the evaporating surface}

The boundary condition for the Euler set of equations on the evaporating surface $\left[\left(\hat{v}_{j}\right)_{b} n_{j}>0\right]$ is given in the following form:

$$
\begin{aligned}
& M_{n} \leqslant 1, \quad M_{t}=0, \\
& \frac{\left(\hat{p}^{A}\right)_{b}}{\hat{p}_{w}^{A}}=h_{1}\left(M_{n}\right), \quad \frac{(\hat{T})_{b}}{\hat{T}_{w}}=h_{2}\left(M_{n}\right) .
\end{aligned}
$$

The functions $h_{1}\left(M_{n}\right)$ and $h_{2}\left(M_{n}\right)$ in Eq. (7b) have been constructed numerically by the use of the Bhatnagar-GrossKrook (BGK) model ${ }^{27-29}$ of the Boltzmann equation in Ref. 23. The analytical form of these functions for $M_{n} \ll 1$ was obtained in Ref. 22 (see also Refs. 5, 24, and 30).

\section{Boundary condition on the condensing surface}

The boundary condition for the Euler set of equations on the condensing surface $\left[\left(\hat{v}_{j}\right)_{b} n_{j}<0\right]$ is summarized as follows:

$$
\begin{aligned}
& \frac{\left(\hat{p}^{A}\right)_{b}}{\hat{p}_{w}^{A}}=\mathcal{F}_{s}\left(M_{n}, M_{t}, \frac{(\hat{T})_{b}}{\hat{T}_{w}}, \frac{l_{r}}{\left(\hat{n}^{A}\right)_{b} l_{b}} \tilde{\Gamma}\right) \quad\left(\text { when } M_{n}<1\right), \\
& \frac{\left(\hat{p}^{A}\right)_{b}}{\hat{p}_{w}^{A}} \geqslant \mathcal{F}_{b}\left(1, M_{t}, \frac{(\hat{T})_{b}}{\hat{T}_{w}}, \frac{l_{r}}{\left(\hat{n}^{A}\right)_{b} l_{b}} \tilde{\Gamma}\right) \quad\left(\text { when } M_{n}=1\right), \\
& \frac{\left(\hat{p}^{A}\right)_{b}}{\hat{p}_{w}^{A}}>\mathcal{F}_{b}\left(M_{n}, M_{t}, \frac{(\hat{T})_{b}}{\hat{T}_{w}}, \frac{l_{r}}{\left(\hat{n}^{A}\right)_{b} l_{b}} \tilde{\Gamma}\right) \quad\left(\text { when } M_{n}>1\right),
\end{aligned}
$$

$\chi_{1,1} \frac{\partial}{\partial \chi_{1}}\left(\hat{\mathcal{N}}_{i}^{B} t_{i}^{(1)}\right)+\chi_{2,2} \frac{\partial}{\partial \chi_{2}}\left(\hat{\mathcal{N}}_{i}^{B} t_{i}^{(2)}\right)+g_{2} \hat{\mathcal{N}}_{i}^{B} t_{i}^{(1)}-g_{1} \hat{\mathcal{N}}_{i}^{B} t_{i}^{(2)}=0$,

$$
\begin{aligned}
\hat{\mathcal{N}}_{i}^{B}= & \hat{v}_{w i} \tilde{\Gamma}+\frac{l_{b}}{l_{r}}\left(\hat{n}^{A}\right)_{b}(\hat{T})_{b}^{1 / 2} \\
& \times G_{s}\left(M_{n}, M_{t}, \frac{(\hat{T})_{b}}{\hat{T}_{w}}, \frac{l_{r}}{\left(\hat{n}^{A}\right)_{b} l_{b}} \widetilde{\Gamma}\right) a_{i} \quad\left(\text { when } M_{n}<1\right),
\end{aligned}
$$

$$
\begin{aligned}
\hat{\mathcal{N}}_{i}^{B}= & \hat{v}_{w i} \widetilde{\Gamma}+\frac{l_{b}}{l_{r}}\left(\hat{n}^{A}\right)_{b}(\hat{T})_{b}^{1 / 2} \\
& \times G_{b}\left(M_{n}, M_{t}, \frac{(\hat{T})_{b}}{\hat{T}_{w}}, \frac{\left(\hat{p}^{A}\right)_{b}}{\hat{p}_{w}^{A}}, \frac{l_{r}}{\left(\hat{n}^{A}\right)_{b} l_{b}} \widetilde{\Gamma}\right) a_{i} \\
& \left(\text { when } M_{n} \geqslant 1\right),
\end{aligned}
$$

where $l_{b}$ is the mean free path of the vapor molecules in the equilibrium state at rest with number density $n_{r}\left(\hat{n}^{A}\right)_{b}$ and temperature $T_{r}(\hat{T})_{b}$ and is therefore a function of $\chi_{1}$ and $\chi_{2}$; $\chi_{1,1}$ and $\chi_{2,2}$ are defined by

$$
\chi_{1,1}=\left(\frac{\partial \chi_{1}}{\partial x_{j}}\right)_{b} t_{j}^{(1)}, \quad \chi_{2,2}=\left(\frac{\partial \chi_{2}}{\partial x_{j}}\right)_{b} t_{j}^{(2)},
$$

and $g_{1}$ and $g_{2}$ are, respectively, the geodesic curvatures ${ }^{31}$ (in the dimensionless $x_{i}$ space) of the $\chi_{1}$ and $\chi_{2}$ coordinate lines on the boundary; and $a_{i}$, which is defined by

$$
a_{i}=\frac{\left(\hat{v}_{i}\right)_{b}-\hat{v}_{w i}-\left(\hat{v}_{j}\right)_{b} n_{j} n_{i}}{\left|\left(\hat{v}_{i}\right)_{b}-\hat{v}_{w i}-\left(\hat{v}_{j}\right)_{b} n_{j} n_{i}\right|}
$$

is the tangential unit vector in the direction of the tangential component of the flow velocity of the vapor relative to the boundary. When the tangential component is zero $\left[\left(\hat{v}_{i}\right)_{b}\right.$ $-\hat{v}_{w i}-\left(\hat{v}_{j}\right)_{b} n_{j} n_{i}=0$ or $M_{t}=0$ ], we can define $a_{i}$ as an arbitrary unit tangential vector because $G_{s}$ and $G_{b}$ vanish [see Eq. (13) below]. Here, we note the following: In Ref. 18, $\left|M_{n}\right|$ instead of $M_{n}$ appears in the equations corresponding to Eqs. (8) and (10) because $M_{n}$ is defined by $M_{n}=\sqrt{6 / 5}(\hat{T})_{b}^{-1 / 2}\left(\hat{v}_{j}\right)_{b} n_{j}$ [cf. Eq. (6a)]; in the same reference, $\mathcal{F}_{s}\left(1_{-}, \cdots\right)$, which is the same as $\mathcal{F}_{b}(1, \cdots)$, is used in the equation corresponding to 
Eq. (8b); and $\hat{\mathcal{N}}_{i 0}$ in Ref. 18 corresponds to $\hat{\mathcal{N}}_{i}^{B}$ here. The $\widetilde{\Gamma}$ and thus $\hat{\mathcal{N}}_{i}^{B}$ are undetermined functions of $\chi_{1}$ and $\chi_{2}$, determined together with the solution of the Euler set of equations. The physical meaning of these functions will be explained below. The functions $\mathcal{F}_{s}$ and $\mathcal{F}_{b}$ of four independent variables occurring in Eq. (8) have been constructed numerically in Refs. 19 and 20 (see also Refs. 25 and 26), on the basis of the model Boltzmann equation proposed by Garzó $e t$ $a l .{ }^{32}$ [the Garzó-Santos-Brey (GSB) model]. It should be mentioned that these numerical functions are restricted to the case where the molecules of the noncondensable gas are mechanically identical to those of the vapor. The numerical data for these functions are given in Refs. 19 and 20: see Eq. (25) in Ref. 19 and related figures and tables for $\mathcal{F}_{s}$; and see Eq. (15a) in Ref. 20 and related figures and tables for $\mathcal{F}_{b}$. The numerical data for the functions $G_{s}$ in Eq. (10a) and $G_{b}$ in Eq. (10b), based on the GSB model and restricted to the case where the molecules of the noncondensable gas and those of the vapor are mechanically the same, are also given in Refs. 19 and 20. More specifically, $G_{s}$ corresponds to $\hat{\mathcal{N}}_{f}=\Gamma \mathcal{G}$ in Ref. 19 [see Eq. (32) there; $\Gamma$ corresponds to $l_{r} \widetilde{\Gamma} /\left(\hat{n}^{A}\right)_{b} l_{b}$ here], and $\mathcal{G}$ is tabulated there (see Fig. 9 and Table III in Ref. 19 and also Tables IX-XII in Ref. 33); and $G_{b}$ corresponds to $\hat{\mathcal{N}}_{f}$ in Ref. 20 (see Fig. 8 and Table III in Ref. 20 and also Tables IX-XII in Ref. 34). We note that the functions $G_{s}$ and $G_{b}$ are nonnegative and have the property that

$$
G_{s}=G_{b}=0 \quad \text { only when } M_{t}=0 \text { or } \tilde{\Gamma}=0 .
$$

When the problem is spatially two dimensional, we may assume that the physical quantities are independent of $\chi_{2}$. For simplicity, let us denote $\chi_{1}$ by $\chi$ and $t_{i}^{(1)}$ by $t_{i}$. Then, because $g_{1}=g_{2}=0$ in this case, it follows from Eq. (9) that

$$
\hat{\mathcal{N}}_{i}^{B} t_{i}=\text { const. }
$$

The functions $\widetilde{\Gamma}$ and $\hat{\mathcal{N}}_{i}^{B}$ of $\chi_{1}$ and $\chi_{2}$ are auxiliary functions in the boundary condition, and their physical meaning is not necessary to obtain the solution of the Euler set of equations with the boundary conditions (7)-(10). However, these functions themselves have clear physical meaning related to the behavior of the noncondensable gas in the Knudsen layer. Let $n_{r} \hat{n}_{K}^{B}$ and $n_{r}\left(2 k T_{r} / m^{A}\right)^{1 / 2} \Phi_{i K}^{B}$ denote, respectively, the number density and the (local) particle flux of the noncondensable gas molecules in the Knudsen layer, and let $\eta$ be the stretched coordinate normal to the boundary defined by $x_{i}=x_{w i}+\epsilon \eta n_{i}$, where $\epsilon=(\sqrt{\pi} / 2) \mathrm{Kn}$ ( $\eta=0$ corresponds to the boundary). Then, $\hat{n}_{K}^{B}$ and $\Phi_{i K}^{B}$ are functions of $\chi_{1}, \chi_{2}$, and $\eta$, and $\widetilde{\Gamma}$ and $\hat{\mathcal{N}}_{i}^{B}$ are defined as ${ }^{18}$

$$
\begin{aligned}
& \tilde{\Gamma}=\int_{0}^{\infty} \hat{n}_{K}^{B} d \eta, \\
& \hat{\mathcal{N}}_{i}^{B}=\int_{0}^{\infty} \Phi_{i K}^{B} d \eta .
\end{aligned}
$$

Thus, $(\sqrt{\pi} / 2) n_{r} l_{r} \widetilde{\Gamma}$ and $(\sqrt{\pi} / 2) n_{r}\left(2 k T_{r} / m^{A}\right)^{1 / 2} l_{r} \hat{\mathcal{N}}_{i}^{B}$ are, respectively, the total number and the total particle flux of the

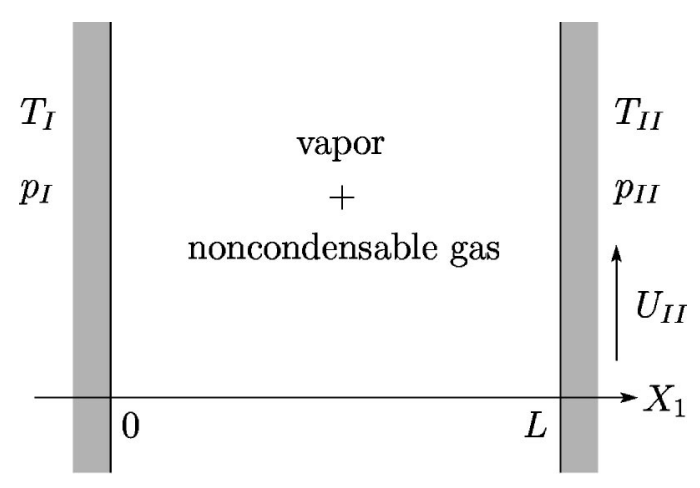

FIG. 1. Two-surface problem of evaporation and condensation in the presence of a noncondensable gas.

noncondensable-gas molecules contained in the Knudsen layer per unit area on the boundary.

\section{Summary}

To summarize, the vapor flows in the continuum limit in the situation explained in Sec. II A are described by the Euler set of equations, Eqs. (5a)-(5d), with the boundary condition, Eqs. (7a) and (7b), when evaporation of the vapor is taking place and with the boundary condition, Eqs. (8a)-(8c), (9), (10a), and (10b), when condensation is taking place. In this physical situation, the noncondensable gas of an infinitesimal average concentration accumulates in the Knudsen layer with an infinitesimal thickness on the condensing boundary. As a result, its local concentration on the boundary rises to the order of unity. In this way, the noncondensable gas has a finite effect on the vapor flow through the boundary condition on the condensing boundary (or more precisely, through $\widetilde{\Gamma}$ in the boundary condition).

\section{APPLICATIONS}

In this section, we show some applications of the Euler system summarized in the preceding section. To be consistent with the numerical data for the functions $\mathcal{F}_{s}, \mathcal{F}_{b}, G_{s}$, and $G_{b}$ given in Refs. 19 and 20 [see Sec. II C for the correspondence between $\left(G_{s}, G_{b}\right)$ and the symbols in Refs. 19 and 20], we assume that the molecules of the noncondensable gas are mechanically identical to those of the vapor and that the behavior of the mixture is described by the GSB model. Then, we make use of these data as well as those for the functions $h_{1}$ and $h_{2}$ based on the BGK model given in Ref. 23.

\section{A. Two-surface problem of evaporation and condensation}

The first problem is the two-surface problem of evaporation and condensation. We consider a vapor (A component) in the gap $0 \leqslant X_{1} \leqslant L$ between two parallel plane condensed phases (see Fig. 1). Let us suppose that the surface at $X_{1}$ $=0$ is kept at temperature $T_{\mathrm{I}}$ and is set at rest, whereas that at $X_{1}=L$ is kept at temperature $T_{\mathrm{II}}$ and may be moving in its surface in the $X_{2}$ direction with a constant speed $U_{\mathrm{II}}$. We denote by $p_{\mathrm{I}}$ and $p_{\mathrm{II}}$ the saturation pressure of the vapor at 
TABLE I. The quantities $\hat{\rho}^{A}, \hat{v}_{1}, \hat{T}, \hat{p}^{A}, M_{n}$, and $\hat{\mathcal{M}}$ for different values of the parameters $p_{\text {II }} / p_{\mathrm{I}}, \hat{U}_{\mathrm{II}}$ and $\Delta\left(T_{\mathrm{II}} / T_{\mathrm{I}}=1\right)$. Here, the molecules of the noncondensable gas are assumed to be mechanically identical with those of the vapor.

\begin{tabular}{|c|c|c|c|c|c|c|c|c|c|c|c|c|c|}
\hline \multirow[b]{2}{*}{$\hat{U}_{\mathrm{II}}$} & \multirow[b]{2}{*}{$\Delta$} & \multicolumn{6}{|c|}{$p_{\mathrm{II}} / p_{\mathrm{I}}=1.2$} & \multicolumn{6}{|c|}{$p_{\mathrm{II}} / p_{\mathrm{I}}=1.5$} \\
\hline & & $\hat{\rho}^{A}$ & $-\hat{v}_{1}$ & $\hat{T}$ & $\hat{p}^{A}$ & $M_{n}$ & $\hat{\mathcal{M}}$ & $\hat{\rho}^{A}$ & $-\hat{v}_{1}$ & $\hat{T}$ & $\hat{p}^{A}$ & $M_{n}$ & $\hat{\mathcal{M}}$ \\
\hline 0 & 0 & 1.118 & 0.0424 & 0.981 & 1.097 & 0.0469 & 0.0474 & 1.284 & 0.0930 & 0.958 & 1.231 & 0.1041 & 0.1194 \\
\hline 0 & 0.5 & 1.128 & 0.0370 & 0.983 & 1.109 & 0.0409 & 0.0417 & 1.312 & 0.0801 & 0.964 & 1.265 & 0.0894 & 0.1051 \\
\hline 0 & 1 & 1.136 & 0.0329 & 0.985 & 1.119 & 0.0363 & 0.0374 & 1.333 & 0.0704 & 0.969 & 1.291 & 0.0783 & 0.0938 \\
\hline 0 & 2 & 1.147 & 0.0269 & 0.988 & 1.134 & 0.0297 & 0.0309 & 1.364 & 0.0567 & 0.975 & 1.329 & 0.0629 & 0.0773 \\
\hline 0.5 & 0 & 1.120 & 0.0414 & 0.981 & 1.099 & 0.0458 & 0.0464 & 1.288 & 0.0912 & 0.959 & 1.235 & 0.1020 & 0.1175 \\
\hline 0.5 & 0.5 & 1.129 & 0.0363 & 0.984 & 1.111 & 0.0401 & 0.0410 & 1.315 & 0.0787 & 0.965 & 1.269 & 0.0878 & 0.1035 \\
\hline 0.5 & 1 & 1.137 & 0.0324 & 0.986 & 1.120 & 0.0357 & 0.0368 & 1.336 & 0.0693 & 0.969 & 1.294 & 0.0771 & 0.0926 \\
\hline 0.5 & 2 & 1.148 & 0.0265 & 0.988 & 1.135 & 0.0292 & 0.0304 & 1.365 & 0.0560 & 0.975 & 1.331 & 0.0621 & 0.0764 \\
\hline 1 & 0 & 1.124 & 0.0388 & 0.983 & 1.105 & 0.0429 & 0.0436 & 1.299 & 0.0861 & 0.961 & 1.249 & 0.0962 & 0.1118 \\
\hline 1 & 0.5 & 1.133 & 0.0343 & 0.985 & 1.116 & 0.0379 & 0.0389 & 1.323 & 0.0749 & 0.967 & 1.279 & 0.0834 & 0.0991 \\
\hline 1 & 1 & 1.140 & 0.0307 & 0.986 & 1.124 & 0.0339 & 0.0350 & 1.342 & 0.0663 & 0.970 & 1.303 & 0.0737 & 0.0890 \\
\hline 1 & 2 & 1.150 & 0.0254 & 0.989 & 1.137 & 0.0280 & 0.0292 & 1.370 & 0.0540 & 0.976 & 1.337 & 0.0598 & 0.0740 \\
\hline 2 & 0 & 1.139 & 0.0312 & 0.986 & 1.123 & 0.0344 & 0.0355 & 1.332 & 0.0708 & 0.968 & 1.290 & 0.0788 & 0.0943 \\
\hline 2 & 0.5 & 1.145 & 0.0282 & 0.987 & 1.131 & 0.0310 & 0.0323 & 1.350 & 0.0629 & 0.972 & 1.312 & 0.0699 & 0.0849 \\
\hline 2 & 1 & 1.150 & 0.0257 & 0.989 & 1.137 & 0.0283 & 0.0296 & 1.364 & 0.0566 & 0.975 & 1.330 & 0.0628 & 0.0772 \\
\hline 2 & 2 & 1.157 & 0.0218 & 0.990 & 1.146 & 0.0240 & 0.0252 & 1.385 & 0.0473 & 0.979 & 1.356 & 0.0524 & 0.0655 \\
\hline
\end{tabular}

temperature $T_{\mathrm{I}}$ and that at temperature $T_{\mathrm{II}}$, respectively. A noncondensable gas ( $B$ component) is contained in the gap, whose average molecular number density over the gap is denoted by $n_{a v}^{B}$. Here, we take $L$ as the reference length and $T_{\mathrm{I}}, p_{\mathrm{I}}$, and $n_{\mathrm{I}}=p_{\mathrm{I}} / k T_{\mathrm{I}}$ as the reference quantities, i.e., $T_{r}=T_{\mathrm{I}}$, $p_{r}=p_{\mathrm{I}}$, and $n_{r}=n_{\mathrm{I}}$. Then, the problem is characterized by the following parameters:

$$
\frac{T_{\mathrm{II}}}{T_{\mathrm{I}}}, \quad \frac{p_{\mathrm{II}}}{p_{\mathrm{I}}}, \quad \hat{U}_{\mathrm{II}}=\frac{U_{\mathrm{II}}}{\left(2 k T_{\mathrm{I}} / m^{A}\right)^{1 / 2}}, \quad \mathrm{Kn}=\frac{l_{r}}{L}, \quad \frac{n_{a v}^{B}}{n_{\mathrm{I}}},
$$

where $l_{r}$ is the mean free path of the vapor molecules in the equilibrium state at rest with temperature $T_{\mathrm{I}}$ and pressure $p_{\mathrm{I}}$ (or molecular number density $n_{\mathrm{I}}$ ). We assume that $T_{\mathrm{I}} \leqslant T_{\mathrm{II}}$ and $p_{\mathrm{I}}<p_{\mathrm{II}}$, so that evaporation takes place on the surface at

TABLE II. The quantities $\hat{\rho}^{A}, \hat{v}_{1}, \hat{T}, \hat{p}^{A}, M_{n}$, and $\hat{\mathcal{M}}$ for different values of the parameters $p_{\mathrm{II}} / p_{\mathrm{I}}, \hat{U}_{\mathrm{II}}$ and $\Delta\left(T_{\mathrm{II}} / T_{\mathrm{I}}=1.1\right)$. See the caption of Table I.

\begin{tabular}{|c|c|c|c|c|c|c|c|c|c|c|c|c|c|}
\hline \multirow[b]{2}{*}{$\hat{U}_{\text {II }}$} & \multirow[b]{2}{*}{$\Delta$} & \multicolumn{6}{|c|}{$p_{\mathrm{II}} / p_{\mathrm{I}}=2$} & \multicolumn{6}{|c|}{$p_{\mathrm{II}} / p_{\mathrm{I}}=5$} \\
\hline & & $\hat{\rho}^{A}$ & $-\hat{v}_{1}$ & $\hat{T}$ & $\hat{p}^{A}$ & $M_{n}$ & $\hat{\mathcal{M}}$ & $\hat{\rho}^{A}$ & $-\hat{v}_{1}$ & $\hat{T}$ & $\hat{p}^{A}$ & $M_{n}$ & $\hat{\mathcal{M}}$ \\
\hline 0 & 0 & 1.402 & 0.1643 & 1.023 & 1.434 & 0.1779 & 0.2303 & 2.600 & 0.3602 & 0.928 & 2.412 & 0.4096 & 0.9365 \\
\hline 0 & 0.5 & 1.457 & 0.1396 & 1.034 & 1.507 & 0.1504 & 0.2033 & 2.923 & 0.2824 & 0.966 & 2.823 & 0.3147 & 0.8255 \\
\hline 0 & 1 & 1.499 & 0.1216 & 1.043 & 1.563 & 0.1305 & 0.1823 & 3.143 & 0.2347 & 0.989 & 3.109 & 0.2585 & 0.7377 \\
\hline 0 & 2 & 1.558 & 0.0969 & 1.054 & 1.643 & 0.1034 & 0.1510 & 3.434 & 0.1775 & 1.016 & 3.490 & 0.1929 & 0.6095 \\
\hline 0.5 & 0 & 1.408 & 0.1615 & 1.024 & 1.442 & 0.1749 & 0.2274 & 2.616 & 0.3559 & 0.930 & 2.433 & 0.4043 & 0.9310 \\
\hline 0.5 & 0.5 & 1.462 & 0.1376 & 1.035 & 1.513 & 0.1482 & 0.2011 & 2.936 & 0.2794 & 0.967 & 2.840 & 0.3112 & 0.8203 \\
\hline 0.5 & 1 & 1.502 & 0.1201 & 1.044 & 1.568 & 0.1287 & 0.1803 & 3.154 & 0.2325 & 0.990 & 3.122 & 0.2560 & 0.7333 \\
\hline 0.5 & 2 & 1.561 & 0.0959 & 1.055 & 1.646 & 0.1023 & 0.1497 & 3.441 & 0.1761 & 1.017 & 3.499 & 0.1913 & 0.6060 \\
\hline 1 & 0 & 1.425 & 0.1540 & 1.027 & 1.464 & 0.1664 & 0.2195 & 2.663 & 0.3440 & 0.936 & 2.492 & 0.3895 & 0.9161 \\
\hline 1 & 0.5 & 1.475 & 0.1319 & 1.038 & 1.530 & 0.1419 & 0.1946 & 2.973 & 0.2711 & 0.971 & 2.888 & 0.3013 & 0.8060 \\
\hline 1 & 1 & 1.513 & 0.1156 & 1.046 & 1.582 & 0.1239 & 0.1749 & 3.184 & 0.2263 & 0.993 & 3.162 & 0.2488 & 0.7205 \\
\hline 1 & 2 & 1.568 & 0.0930 & 1.056 & 1.656 & 0.0991 & 0.1458 & 3.462 & 0.1723 & 1.019 & 3.527 & 0.1870 & 0.5965 \\
\hline 2 & 0 & 1.478 & 0.1304 & 1.039 & 1.535 & 0.1401 & 0.1927 & 2.822 & 0.3054 & 0.955 & 2.694 & 0.3424 & 0.8618 \\
\hline 2 & 0.5 & 1.517 & 0.1139 & 1.046 & 1.588 & 0.1219 & 0.1728 & 3.098 & 0.2441 & 0.984 & 3.050 & 0.2695 & 0.7562 \\
\hline 2 & 1 & 1.547 & 0.1013 & 1.052 & 1.628 & 0.1082 & 0.1567 & 3.285 & 0.2060 & 1.003 & 3.294 & 0.2253 & 0.6767 \\
\hline 2 & 2 & 1.592 & 0.0833 & 1.061 & 1.689 & 0.0886 & 0.1327 & 3.532 & 0.1593 & 1.025 & 3.620 & 0.1724 & 0.5626 \\
\hline
\end{tabular}


TABLE III. The quantities $\hat{\rho}^{A}, \hat{v}_{1}, \hat{T}, \hat{p}^{A}, M_{n}$, and $\hat{\mathcal{M}}$ for different values of the parameters $p_{\text {II }} / p_{\mathrm{I}}, \hat{U}_{\mathrm{II}}$ and $\Delta\left(T_{\mathrm{II}} / T_{\mathrm{I}}=1.2\right)$. See the caption of Table I.

\begin{tabular}{|c|c|c|c|c|c|c|c|c|c|c|c|c|c|}
\hline \multirow[b]{2}{*}{$\hat{U}_{\mathrm{II}}$} & \multirow[b]{2}{*}{$\Delta$} & \multicolumn{6}{|c|}{$p_{\mathrm{II}} / p_{\mathrm{I}}=5$} & \multicolumn{6}{|c|}{$p_{\mathrm{II}} / p_{\mathrm{I}}=10$} \\
\hline & & $\hat{\rho}^{A}$ & $-\hat{v}_{1}$ & $\hat{T}$ & $\hat{p}^{A}$ & $M_{n}$ & $\hat{\mathcal{M}}$ & $\hat{\rho}^{A}$ & $-\hat{v}_{1}$ & $\hat{T}$ & $\hat{p}^{A}$ & $M_{n}$ & $\hat{\mathcal{M}}$ \\
\hline 0 & 0 & 2.381 & 0.3768 & 1.012 & 2.409 & 0.4103 & 0.8972 & 3.929 & 0.5136 & 0.940 & 3.694 & 0.5803 & 2.018 \\
\hline 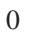 & 0.5 & 2.665 & 0.2986 & 1.052 & 2.803 & 0.3190 & 0.7958 & 4.754 & 0.3779 & 1.011 & 4.808 & 0.4117 & 1.797 \\
\hline & 1 & 2.862 & 0.2497 & 1.077 & 3.081 & 0.2636 & 0.7146 & 5.271 & 0.3063 & 1.048 & 5.523 & 0.3278 & 1.615 \\
\hline 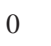 & 2 & 3.125 & 0.1902 & 1.106 & 3.458 & 0.1980 & 0.5943 & 5.924 & 0.2264 & 1.088 & 6.447 & 0.2377 & 1.341 \\
\hline 0.5 & 0 & 2.395 & 0.3726 & 1.014 & 2.429 & 0.4053 & 0.8924 & 3.954 & 0.5090 & 0.942 & 3.727 & 0.5744 & 2.013 \\
\hline 0.5 & 0.5 & 2.677 & 0.2956 & 1.053 & 2.819 & 0.3155 & 0.7913 & 4.775 & 0.3748 & 1.013 & 4.837 & 0.4079 & 1.789 \\
\hline 0.5 & 1 & 2.872 & 0.2475 & 1.078 & 3.095 & 0.2611 & 0.7108 & 5.289 & 0.3040 & 1.049 & 5.548 & 0.3251 & 1.608 \\
\hline 0.5 & 2 & 3.132 & 0.1887 & 1.107 & 3.467 & 0.1965 & 0.5910 & 5.937 & 0.2249 & 1.089 & 6.465 & 0.2361 & 1.335 \\
\hline 1 & 0 & 2.437 & 0.3606 & 1.020 & 2.486 & 0.3910 & 0.8788 & 4.025 & 0.4962 & 0.949 & 3.822 & 0.5578 & 1.997 \\
\hline 1 & 0.5 & 2.710 & 0.2871 & 1.058 & 2.866 & 0.3058 & 0.7780 & 4.836 & 0.3659 & 1.018 & 4.921 & 0.3974 & 1.769 \\
\hline 1 & 1 & 2.899 & 0.2411 & 1.081 & 3.133 & 0.2540 & 0.6989 & 5.340 & 0.2973 & 1.052 & 5.620 & 0.3175 & 1.588 \\
\hline 1 & 2 & 3.151 & 0.1847 & 1.109 & 3.494 & 0.1921 & 0.5820 & 5.973 & 0.2208 & 1.091 & 6.517 & 0.2315 & 1.319 \\
\hline 2 & 0 & 2.578 & 0.3215 & 1.040 & 2.681 & 0.3453 & 0.8288 & 4.273 & 0.4534 & 0.972 & 4.153 & 0.5037 & 1.937 \\
\hline 2 & 0.5 & 2.822 & 0.2594 & 1.072 & 3.024 & 0.2745 & 0.7320 & 5.042 & 0.3368 & 1.032 & 5.206 & 0.3631 & 1.698 \\
\hline 2 & 1 & 2.990 & 0.2200 & 1.091 & 3.263 & 0.2307 & 0.6578 & 5.513 & 0.2754 & 1.064 & 5.863 & 0.2926 & 1.518 \\
\hline 2 & 2 & 3.215 & 0.1712 & 1.116 & 3.587 & 0.1775 & 0.5504 & 6.097 & 0.2069 & 1.098 & 6.694 & 0.2163 & 1.261 \\
\hline
\end{tabular}

$X_{1}=L\left(\right.$ or $\left.x_{1}=1\right)$ and condensation at $X_{1}=0$ (or $\left.x_{1}=0\right)$; therefore $\hat{v}_{1}<0$. In view of Eq. (1), we let

$$
\frac{n_{a v}^{B}}{n_{\mathrm{I}}}=\Delta \mathrm{Kn},
$$

where $\Delta$ is a constant, and we specify $\Delta$ rather than $n_{a v}^{B} / n_{\mathrm{I}}$. We investigate the steady flow of the vapor caused by evaporation and condensation on the condensed phases and the effect of the noncondensable gas on the vapor flow in the continuum limit with respect to the vapor $(\mathrm{Kn} \rightarrow 0)$. The present problem has been investigated in Ref. 13 in the case of $\hat{U}_{\mathrm{II}}=0$. The following analysis and discussion are straightforward extensions of those given there.

Now, let us apply the Euler equations and their boundary conditions to the present problem. Since the problem is spatially one-dimensional, $\widetilde{\Gamma}$ in Eqs. (8a)-(8c) is a constant, which is related to $\Delta$ by $^{13}$

$$
\Delta=\frac{\sqrt{\pi}}{2} \tilde{\Gamma} .
$$

This relation is derived from the following fact: since there is no noncondensable gas except in the Knudsen layer at the condensing surface $\left(x_{1}=0\right)$, the quantity $(\sqrt{\pi} / 2) n_{\mathrm{I}} l_{r} \widetilde{\Gamma}$, which is the total number of the noncondensable-gas molecules contained in the Knudsen layer per unit area of the condensing surface [see the paragraph containing Eq. (15)], is equal to $n_{a v}^{B} L$, i.e., the total number of the noncondensable-gas molecules contained in the column of length $L$ between two condensed phases, perpendicular to them, with a unit base area. In the present one-dimensional problem, $\hat{\mathcal{N}}_{i}^{B}$ $=\left(0, \hat{\mathcal{N}}_{2}^{B}, 0\right)$ is also constant, so that Eq. (9) or Eq. (14) is satisfied.
It follows from Eqs. (5a)-(5d) that $\hat{\rho}^{A}\left(=\hat{n}^{A}\right), \hat{v}_{i}\left(\hat{v}_{3}=0\right)$, $\hat{T}$, and $\hat{p}^{A}$ are all constant. The boundary condition on the evaporating surface (7) leads to the following condition at $x_{1}=1$ :

$$
\begin{aligned}
& M_{n} \leqslant 1, \quad \hat{v}_{2}=\hat{U}_{\mathrm{II}}, \\
& \hat{p}^{A}=\frac{p_{\mathrm{II}}}{p_{\mathrm{I}}} h_{1}\left(M_{n}\right), \quad \hat{T}=\frac{T_{\mathrm{II}}}{T_{\mathrm{I}}} h_{2}\left(M_{n}\right),
\end{aligned}
$$

where

$$
M_{n}=\sqrt{6 / 5} \hat{T}^{-1 / 2}\left|\hat{v}_{1}\right|
$$

The notation $(\cdot)_{b}$ is omitted in Eqs. (19) and (20) as well as in Eqs. (21) and (22) below because $\hat{p}^{A}, \hat{T}$, etc., are constants. The boundary condition on the condensing surface (8)-(10) reduces to the following condition at $x_{1}=0$ :

$$
\begin{aligned}
& \hat{p}^{A}=\mathcal{F}_{s}\left(M_{n}, M_{t}, \hat{T}, \frac{l_{r}}{\hat{n}^{A} l_{b}} \tilde{\Gamma}\right) \quad\left(M_{n}<1\right), \\
& \hat{p}^{A} \geqslant \mathcal{F}_{b}\left(1, M_{t}, \hat{T}, \frac{l_{r}}{\hat{n}^{A} l_{b}} \tilde{\Gamma}\right) \quad\left(M_{n}=1\right), \\
& \hat{p}^{A}>\mathcal{F}_{b}\left(M_{n}, M_{t}, \hat{T}, \frac{l_{r}}{\hat{n}^{A} l_{b}} \tilde{\Gamma}\right) \quad\left(M_{n}>1\right),
\end{aligned}
$$

where $M_{n}$ is the same as Eq. (20), $M_{t}$ is given by

$$
M_{t}=\sqrt{6 / 5} \hat{T}^{-1 / 2}\left|\hat{v}_{2}\right|,
$$

and $l_{b}$ is the mean free path of the vapor molecules in the equilibrium state at rest with temperature $T_{\mathrm{I}} \hat{T}$ and number density $n_{\mathrm{I}} \hat{n}^{A}$. The ratio $l_{r} / l_{b}$, appearing in the functions $\mathcal{F}_{s}$ 
and $\mathcal{F}_{b}$, depends on the molecular model: $l_{r} / l_{b}=\hat{n}^{A} / \hat{T}^{1 / 2}$ for the GSB model (for example, $l_{r} / l_{b}=\hat{n}^{A}$ for hard-sphere molecules).

From the first equation of Eq. (19a), the flow is either subsonic $\left(M_{n}<1\right)$ or sonic $\left(M_{n}=1\right)$. First, we consider the subsonic case. If we eliminate $\hat{p}^{A}, \hat{T}, \hat{v}_{2}$, and $\widetilde{\Gamma}$ from Eqs. (18), (19a), (19b), (21a), and (22), we obtain the following equation for $M_{n}$ :

$$
\begin{aligned}
\frac{p_{\mathrm{II}}}{p_{\mathrm{I}}} h_{1}\left(M_{n}\right)= & \mathcal{F}_{s}\left(M_{n},\left(\frac{6}{5}\right)^{1 / 2} \hat{U}_{\mathrm{II}}\left[\frac{T_{\mathrm{II}}}{T_{\mathrm{I}}} h_{2}\left(M_{n}\right)\right]^{-1 / 2},\right. \\
& \left.\frac{T_{\mathrm{II}}}{T_{\mathrm{I}}} h_{2}\left(M_{n}\right), \frac{2}{\sqrt{\pi}}\left[\frac{T_{\mathrm{II}}}{T_{\mathrm{I}}} h_{2}\left(M_{n}\right)\right]^{-1 / 2} \Delta\right) .
\end{aligned}
$$

The $M_{n}$ is determined by solving Eq. (23) for a given set of the parameter $\left(T_{\mathrm{II}} / T_{\mathrm{I}}, p_{\mathrm{II}} / p_{\mathrm{I}}, \hat{U}_{\mathrm{II}}, \Delta\right)$. Then, $\hat{p}^{A}$ and $\hat{T}$ are obtained from Eq. (19b), $\hat{v}_{1}$ from Eq. (20), and $\hat{\rho}^{A}\left(=\hat{n}^{A}\right)$ from Eq. (5d). We show the constants $\hat{\rho}^{A}, \hat{v}_{1}, \hat{T}, \hat{p}^{A}$, and $M_{n}$ thus obtained for various values of the set $\left(T_{\mathrm{II}} / T_{\mathrm{I}}, p_{\mathrm{II}} / p_{\mathrm{I}}, \hat{U}_{\mathrm{II}}, \Delta\right)$ in Tables I-III. The results for the case $\hat{U}_{\mathrm{II}}=0$ are given in Table I of Ref. 13, where the parameter $n_{\mathrm{II}} / n_{\mathrm{I}}\left(n_{\mathrm{II}}=p_{\mathrm{II}} / k T_{\mathrm{II}}\right)$ is used rather than $p_{\mathrm{II}} / p_{\mathrm{I}}$. In the present study, we recomputed the case of $\hat{U}_{\text {II }}=0$ using supplemented numerical data of $\mathcal{F}_{s}$ and included the results in Tables I-III for the sake of completeness. Since the dependence of $\mathcal{F}_{s}$ on $M_{t}$ is weak, the solution does not depend much on $\hat{U}_{\mathrm{II}}$. Its dependence on $\Delta$ is stronger. The dimensionless mass-flow rate $\hat{\mathcal{M}}\left(=\hat{\rho}^{A}\left|\hat{v}_{1}\right|\right)$ from the evaporating to the condensing surface, which is related to the (dimensional) mass-flow rate $\mathcal{M}\left(=\rho^{A}\left|v_{1}\right|\right)$ per unit time and unit area (through a plane $x_{1}=$ const) as $\mathcal{M}$ $=2 p_{\mathrm{I}}\left(2 k T_{\mathrm{I}} / m^{A}\right)^{-1 / 2} \hat{\mathcal{M}}$, is also shown in Tables I-III. With the increase of $\Delta$ (or the amount of the noncondensable gas in the Knudsen layer at $x_{1}=0$ ), the condensation becomes weaker, and thus $\left|\hat{v}_{1}\right|$ and $\hat{\mathcal{M}}$ decrease. On the other hand, $\hat{v}_{2}=\hat{U}_{\text {II }}$ holds irrespective of $\Delta$. Therefore, when $\hat{U}_{\text {II }}$ is finite (e.g., $\hat{U}_{\mathrm{II}}=0.5,1,2$ in Tables I-III), the flow becomes more tangential to the plane condensed phases for larger $\Delta$. We will see the same effect in Figs. 5 and 6 in Sec. III B.

Next, we consider the case where the flow speed is sonic $\left(M_{n}=1\right)$. If we eliminate $\hat{p}^{A}, \hat{T}, \hat{v}_{2}$, and $\widetilde{\Gamma}$ from Eqs. (18), (19a), (19b), (21b), and (22), we obtain the following relation:

$$
\frac{p_{\mathrm{II}}}{p_{\mathrm{I}}} \geqslant \mathcal{S}\left(\hat{U}_{\mathrm{II}}, \frac{T_{\mathrm{II}}}{T_{\mathrm{I}}}, \Delta\right),
$$

where

$$
\begin{aligned}
\mathcal{S}\left(\hat{U}_{\mathrm{II}}, \frac{T_{\mathrm{II}}}{T_{\mathrm{I}}}, \Delta\right)= & \frac{1}{h_{1}(1)} \mathcal{F}_{b}\left(1,\left(\frac{6}{5}\right)^{1 / 2} \hat{U}_{\mathrm{II}}\left[\frac{T_{\mathrm{II}}}{T_{\mathrm{I}}} h_{2}(1)\right]^{-1 / 2},\right. \\
& \left.\frac{T_{\mathrm{II}}}{T_{\mathrm{I}}} h_{2}(1), \frac{2}{\sqrt{\pi}}\left[\frac{T_{\mathrm{II}}}{T_{\mathrm{I}}} h_{2}(1)\right]^{-1 / 2} \Delta\right) .
\end{aligned}
$$

This relation gives the condition for $T_{\mathrm{II}} / T_{\mathrm{I}}, p_{\mathrm{II}} / p_{\mathrm{I}}, \hat{U}_{\mathrm{II}}$, and $\Delta$ for which a sonic flow $\left(M_{n}=1\right)$ occurs. Some examples of the boundary $\mathcal{S}\left(\hat{U}_{\mathrm{II}}, T_{\mathrm{II}} / T_{\mathrm{I}}, \Delta\right)$ of the existence range (24a)

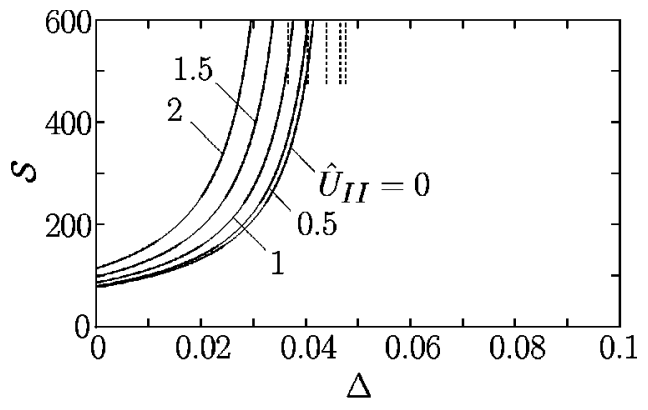

(a) $T_{I I} / T_{I}=1$

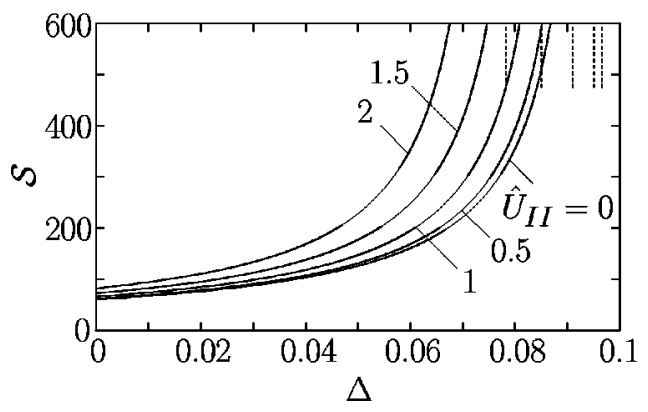

(b) $T_{I I} / T_{I}=2$

FIG. 2. The boundary $\mathcal{S}\left(\hat{U}_{\mathrm{II}}, T_{\mathrm{II}} / T_{\mathrm{I}}, \Delta\right)$ of the existence range vs $\Delta$ for various values of $\hat{U}_{\mathrm{II}}$. (a) $T_{\mathrm{II}} / T_{\mathrm{I}}=1$, (b) $T_{\mathrm{II}} / T_{\mathrm{I}}=2$. The dotted lines in the figures indicate the asymptotes $\left(\Delta=\delta_{c}\right)$ of the curves. Here, the molecules of the noncondensable gas are assumed to be mechanically identical with those of the vapor.

are shown in Fig. 2, where $\mathcal{S}\left(\hat{U}_{\mathrm{II}}, T_{\mathrm{II}} / T_{\mathrm{I}}, \Delta\right)$ is shown as a function of $\Delta$ for $T_{\mathrm{II}} / T_{\mathrm{I}}=1$ and 2 and for various values of $\hat{U}_{\mathrm{II}}$. The $\mathcal{S}\left(\hat{U}_{\mathrm{II}}, T_{\mathrm{II}} / T_{\mathrm{I}}, \Delta\right)$ is an increasing function of $\Delta$ and becomes infinitely large as $\Delta$ approaches a critical value $\delta_{c}$ that depends on $\hat{U}_{\mathrm{II}}$ and $T_{\mathrm{II}} / T_{\mathrm{I}}$. That is, $\Delta=\delta_{c}$ is the asymptote of the curve, and there is no sonic solution in the region $\Delta \geqslant \delta_{c}$. The formula of the critical value $\delta_{c}$ is readily obtained from the form of $\mathcal{F}_{b}$ given in Ref. 20, i.e.,

$$
\begin{aligned}
\delta_{c}= & \frac{\sqrt{\pi}}{2}\left[\frac{T_{\mathrm{II}}}{T_{\mathrm{I}}} h_{2}(1)\right]^{1 / 2} \\
& \times \Gamma_{b}\left(1,\left(\frac{6}{5}\right)^{1 / 2} \hat{U}_{\mathrm{II}}\left[\frac{T_{\mathrm{II}}}{T_{\mathrm{I}}} h_{2}(1)\right]^{-1 / 2}, \frac{T_{\mathrm{II}}}{T_{\mathrm{I}}} h_{2}(1)\right),
\end{aligned}
$$

where $\Gamma_{b}$ is a function the numerical data of which are given

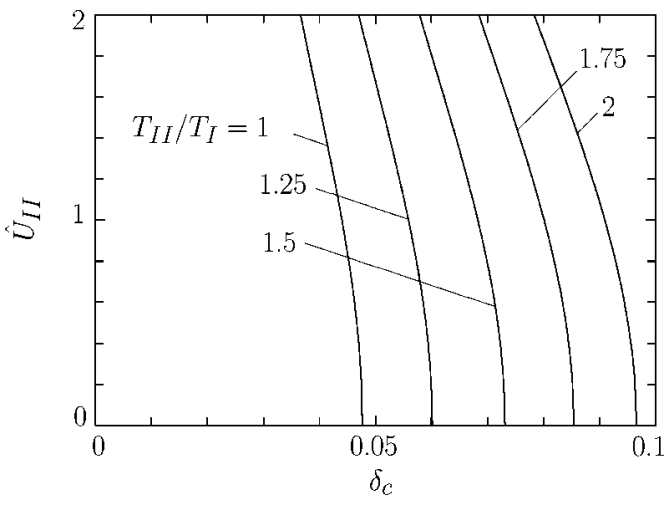

FIG. 3. The critical value $\delta_{c}$ vs $\hat{U}_{\mathrm{II}}$ for various $T_{\mathrm{II}} / T_{\mathrm{I}}$. The $\delta_{c}$ is taken as the abscissa for easy comparison with Fig. 2. See the caption of Fig. 2. 


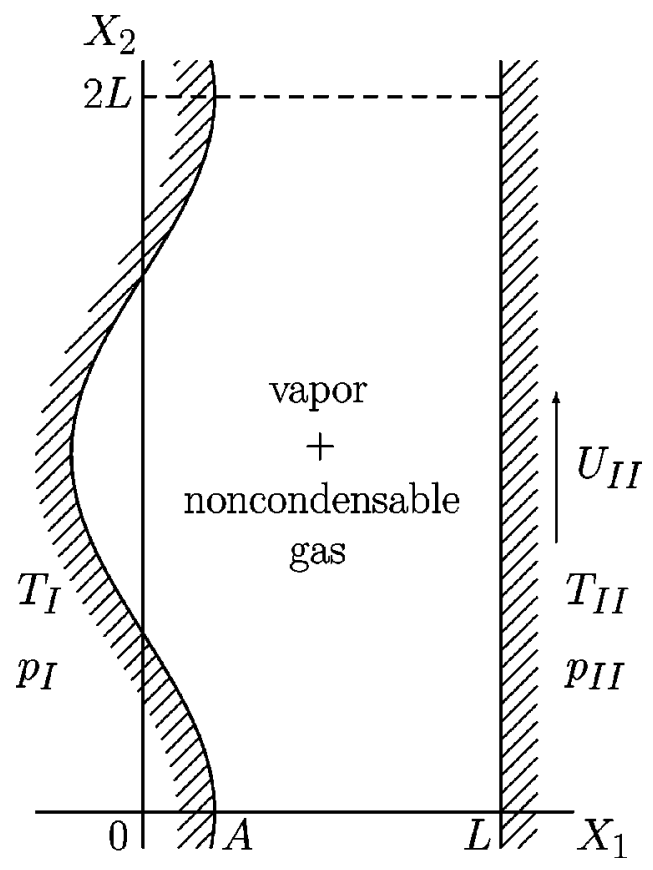

FIG. 4. Vapor flow between a plane and a sinusoidal condensed phase in the presence of a noncondensable gas.

in Ref. 20. In Fig. 3, $\delta_{c}$ versus $\hat{U}_{\mathrm{II}}$ is shown for various values of $T_{\mathrm{II}} / T_{\mathrm{I}} ; \delta_{c}$ is taken as the abscissa for easy comparison with Fig. 2.

Here, we should recall that, because of Eq. (17), $n_{a v}^{B} / n_{\mathrm{I}}$ vanishes in the continuum limit $\mathrm{Kn} \rightarrow 0$. As we have seen, however, the solution in the continuum limit depends on $\Delta$, which is a quantity specifying the vanishing rate of $n_{a v}^{B} / n_{\mathrm{I}}$ and thus related to the amount of the noncondensable gas. This means that, in the continuum limit, the vapor flow is still affected by the noncondensable gas in spite of the fact that its average concentration is infinitesimal.

\section{B. Vapor flows between a plane and a sinusoidal condensed phase}

Next we consider a vapor ( $A$ component) and a noncondensable gas ( $B$ component) in the gap between a sinusoidal condensed phase located at $X_{1}=A \cos \left(\pi X_{2} / L\right)$ and a plane condensed phase located at $X_{1}=L$ (see Fig. 4). Let us suppose that the sinusoidal condensed phase is kept at temperature $T_{\mathrm{I}}$ and is set at rest, whereas the plane condensed phase is kept at temperature $T_{\mathrm{II}}$ and may be moving in its surface in the $X_{2}$ direction with a constant speed $U_{\mathrm{II}}$. We denote by $p_{\mathrm{I}}$ the saturation pressure of the vapor at temperature $T_{\mathrm{I}}$ and by $p_{\text {II }}$ that at temperature $T_{\text {II }}$. We take $L$ as the reference length and $T_{\mathrm{I}}, p_{\mathrm{I}}$, and $n_{\mathrm{I}}=p_{\mathrm{I}} / k T_{\mathrm{I}}$ as the reference quantities, i.e., $T_{r}=T_{\mathrm{I}}, p_{r}=p_{\mathrm{I}}$, and $n_{r}=n_{\mathrm{I}}$. Then, the Knudsen number Kn of the system is given by $\mathrm{Kn}=l_{r} / L$, where $l_{r}$ is the mean free path of the vapor molecules in the equilibrium state at rest with temperature $T_{\mathrm{I}}$ and pressure $p_{\mathrm{I}}$ (or molecular number density $n_{\mathrm{I}}$ ). We assume that the flow field is periodic in the $X_{2}$ direction with period $2 L$ and consider the problem in the closed domain

$$
A \cos \left(\frac{\pi X_{2}}{L}\right) \leqslant X_{1} \leqslant L, \quad 0 \leqslant X_{2} \leqslant 2 L .
$$

We denote by $n_{a v}^{B}$ the average molecular number density of the noncondensable gas over the domain (26) and let it be in the form of Eq. (17), i.e., $n_{a v}^{B} / n_{\mathrm{I}}=\Delta \mathrm{Kn}$, in order to be consistent with Eq. (1). The problem is then characterized by the following parameters:

$$
\frac{T_{\mathrm{II}}}{T_{\mathrm{I}}}, \quad \frac{p_{\mathrm{II}}}{p_{\mathrm{I}}}, \quad \hat{U}_{\mathrm{II}}=\frac{U_{\mathrm{II}}}{\left(2 k T_{\mathrm{I}} / m^{A}\right)^{1 / 2}}, \quad \frac{A}{L}, \quad \mathrm{Kn}, \quad \Delta .
$$

We investigate the steady behavior of the vapor and the noncondensable gas in the continuum limit $(\mathrm{Kn} \rightarrow 0)$, where the average concentration of the noncondensable gas becomes infinitesimal $\left(n_{a v}^{B} / n_{\mathrm{I}} \rightarrow 0\right)$.

We restrict ourselves to the case where evaporation takes place on the plane condensed phase and condensation on the sinusoidal one. In addition, we assume, just for simplicity, that the speed of condensation on the sinusoidal condensed phase is subsonic. The present problem has been investigated on the basis of the same Euler system in Ref. 18 in the case where the plane condensed phase is at rest $\left(\hat{U}_{\mathrm{II}}=0\right)$. This case will also be included in the following discussions.

Now let us summarize the boundary conditions for the Euler set of equations (5a)-(5d) in the present problem. The boundary condition on the plane condensed phase, where evaporation is assumed to be taking place, is given by Eq. (7), i.e., at $x_{1}=1$,

$$
\begin{aligned}
& M_{n} \leqslant 1, \quad \hat{v}_{2}=\hat{U}_{\mathrm{II}}, \\
& \hat{p}^{A}=\frac{p_{\mathrm{II}}}{p_{\mathrm{I}}} h_{1}\left(M_{n}\right), \quad \hat{T}=\frac{T_{\mathrm{II}}}{T_{\mathrm{I}}} h_{2}\left(M_{n}\right),
\end{aligned}
$$

with

$$
M_{n}=\sqrt{6 / 5} \hat{T}^{-1 / 2}\left|\hat{v}_{1}\right| .
$$

In Eqs. (28) and (29) and in Eqs. (30)-(33) below, the notation $(\cdot)_{b}$ is omitted because it is obvious that the quantities occurring in the boundary conditions are their boundary values. The boundary condition on the sinusoidal condensed phase, where subsonic condensation is assumed to be taking place, is given by Eqs. (8a) and (9). That is, at $x_{1}$ $=(A / L) \cos \left(\pi x_{2}\right)$,

$$
\hat{p}^{A}=\mathcal{F}_{s}\left(M_{n}, M_{t}, \hat{T}, \frac{l_{r}}{\hat{n}^{A} l_{b}} \tilde{\Gamma}\right),
$$

where $M_{n}(<1)$ and $M_{t}$ are given by

$$
\begin{aligned}
& M_{n}=\sqrt{6 / 5} \hat{T}^{-1 / 2}\left|\hat{v}_{j} n_{j}\right|, \\
& M_{t}=\sqrt{6 / 5} \hat{T}^{-1 / 2}\left|\hat{v}_{j} t_{j}\right|,
\end{aligned}
$$

with $n_{i}$ and $t_{i}$ being unit normal and tangential vectors to the sinusoidal condensed phase, and $l_{b}$ the mean free path of the molecules of the vapor in the equilibrium state at rest with temperature $T_{\mathrm{I}} \hat{T}$ and number density $n_{\mathrm{I}} \hat{n}^{A}$. In addition, in the present two-dimensional problem, Eq. (14) holds along the sinusoidal boundary with $\hat{\mathcal{N}}_{i}^{B}$ given by [see Eq. (10a)] 


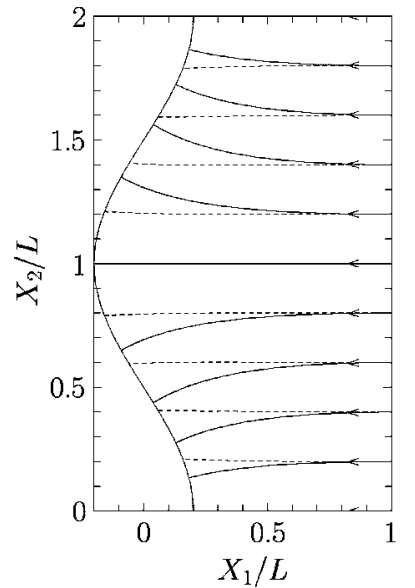

(a) $\hat{U}_{I I}=0$

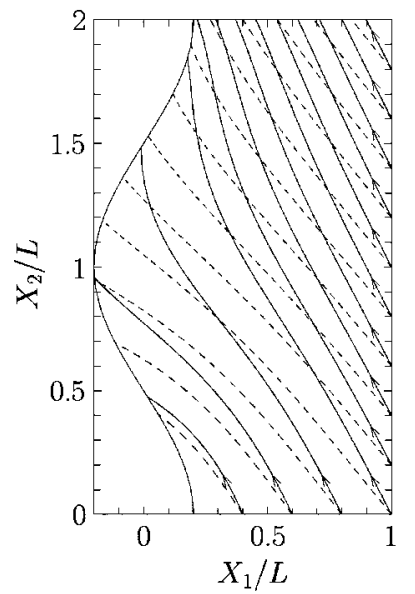

(c) $\hat{U}_{I I}=0.2$

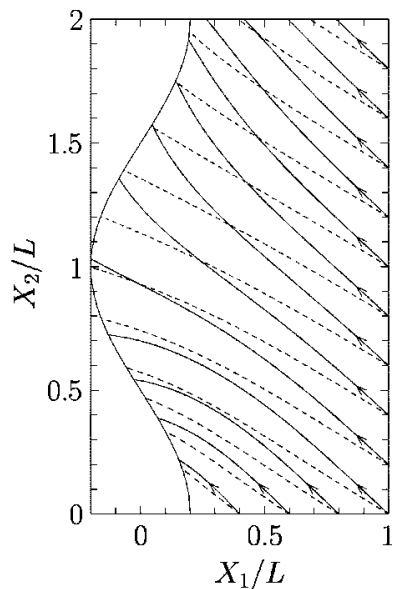

(b) $\hat{U}_{I I}=0.1$

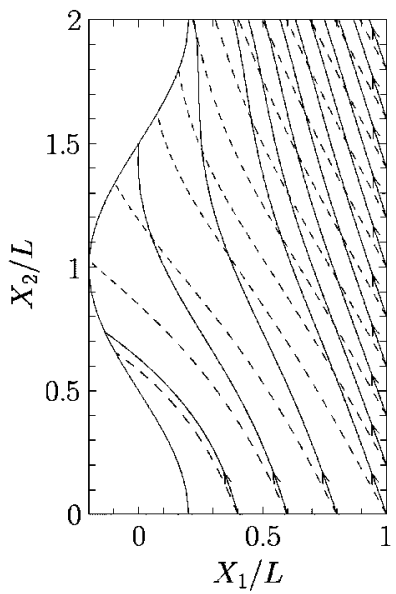

(d) $\hat{U}_{I I}=0.3$

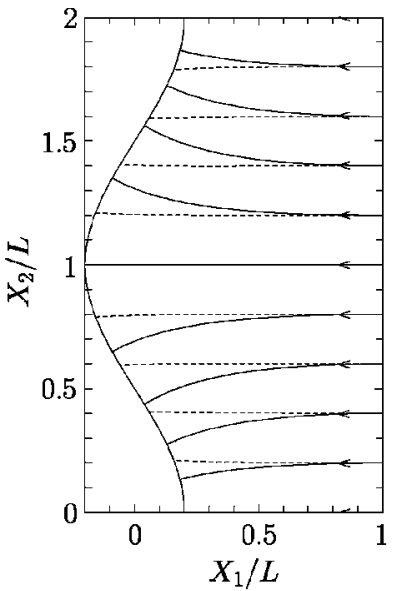

(a) $\hat{U}_{I I}=0$

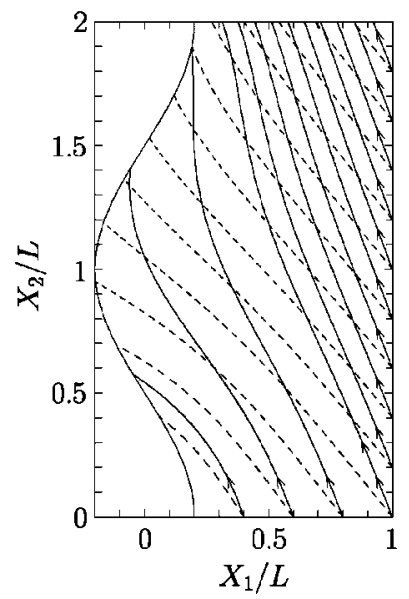

(c) $\hat{U}_{I I}=0.2$

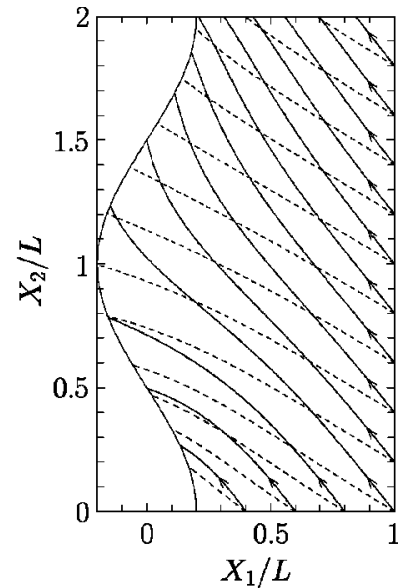

(b) $\hat{U}_{I I}=0.1$

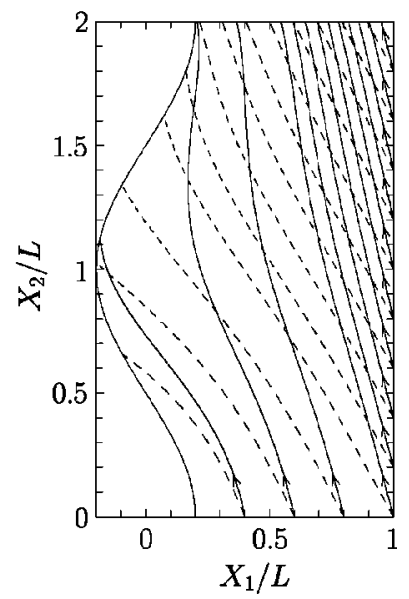

(d) $\hat{U}_{I I}=0.3$

FIG. 5. Streamlines of the vapor flow for $\Delta=2$ in the case of $T_{\mathrm{II}} / T_{\mathrm{I}}=1$, $p_{\mathrm{II}} / p_{\mathrm{I}}=2$, and $A / L=0.2$. (a) $\hat{U}_{\mathrm{II}}=0$, (b) $\hat{U}_{\mathrm{II}}=0.1$, (c) $\hat{U}_{\mathrm{II}}=0.2$, (d) $\hat{U}_{\mathrm{II}}=0.3$. The corresponding streamlines in the pure-vapor case $(\Delta=0)$ are shown by dashed lines. Here, the molecules of the noncondensable gas are assumed to be mechanically identical with those of the vapor.

FIG. 6. Streamlines of the vapor flow for $\Delta=4$ in the case of $T_{\mathrm{II}} / T_{\mathrm{I}}=1$, $p_{\mathrm{II}} / p_{\mathrm{I}}=2$, and $A / L=0.2$. (a) $\hat{U}_{\mathrm{II}}=0$, (b) $\hat{U}_{\mathrm{II}}=0.1$, (c) $\hat{U}_{\mathrm{II}}=0.2$, (d) $\hat{U}_{\mathrm{II}}=0.3$. The corresponding streamlines in the pure-vapor case $(\Delta=0)$ are shown by dashed lines. See the caption of Fig. 5.

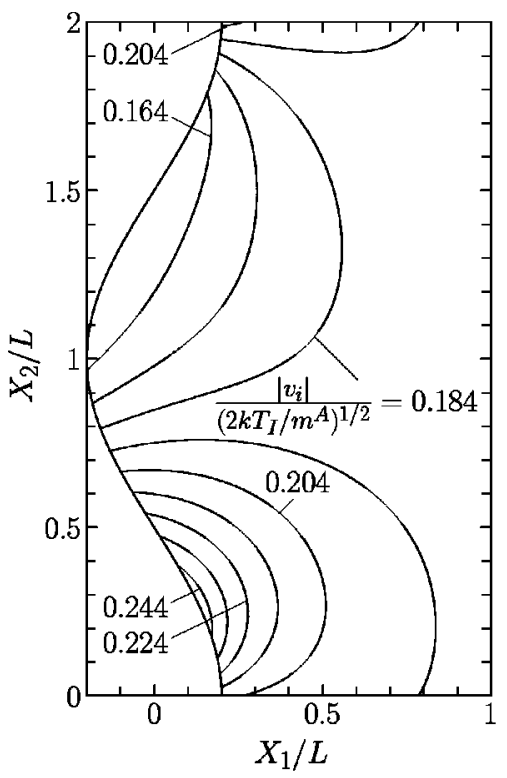

(a) pure vapor $(\Delta=0)$

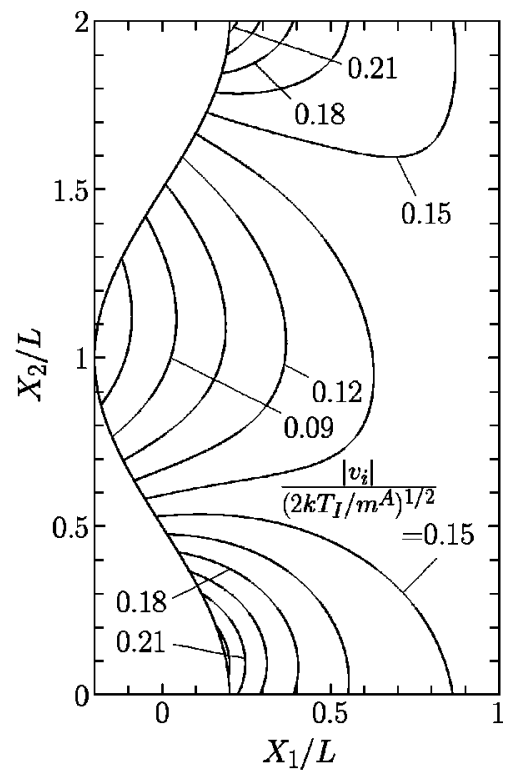

(b) $\Delta=2$
FIG. 7. Isolines of the flow speed $\left|v_{i}\right|$ for $\hat{U}_{\mathrm{II}}=0.1$, $T_{\mathrm{II}} / T_{\mathrm{I}}=1, p_{\mathrm{II}} / p_{\mathrm{I}}=2$, and $A / L=0.2$. (a) Pure vapor $(\Delta$ $=0),\left|v_{i}\right| /\left(2 k T_{\mathrm{I}} / m^{A}\right)^{1 / 2}=0.164+0.01 \mathrm{~m}(m=0, \ldots 8)$; (b) $\Delta=2,\left|v_{i}\right| /\left(2 k T_{\mathrm{I}} / m^{A}\right)^{1 / 2}=0.075+0.015 m(m=0, \ldots, 10)$. See the caption of Fig. 5. 


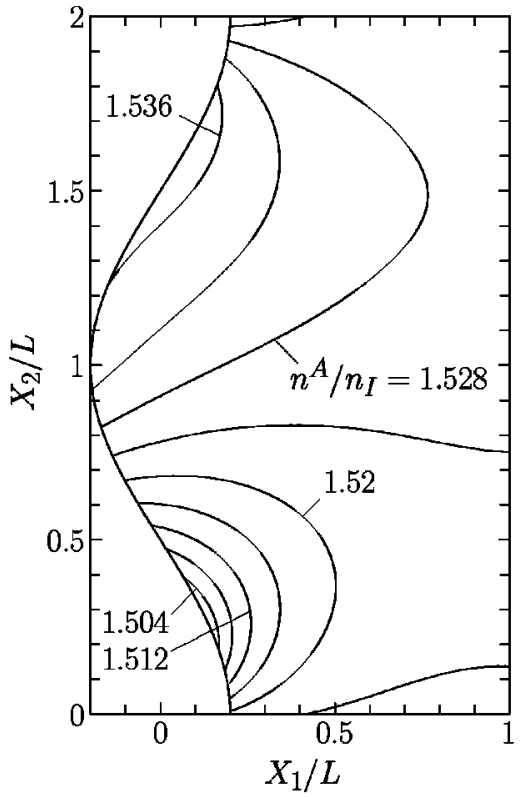

(a) pure vapor $(\Delta=0)$

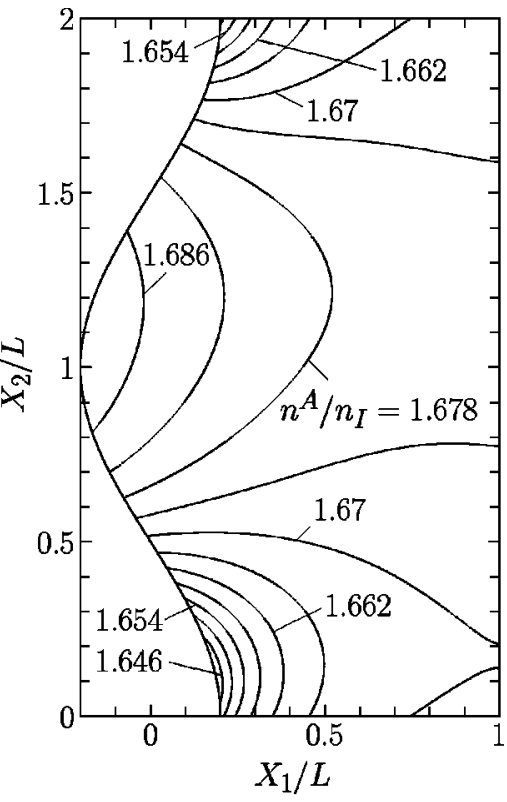

(b) $\Delta=2$
FIG. 8. Isolines of the number density $n^{A}$ for $\hat{U}_{\mathrm{II}}=0.1$, $T_{\mathrm{II}} / T_{\mathrm{I}}=1, p_{\mathrm{II}} / p_{\mathrm{I}}=2$, and $A / L=0.2$. (a) Pure vapor $(\Delta$ $=0), \quad n^{A} / n_{\mathrm{I}}=1.504+0.004 m(m=0, \ldots 8) ; \quad$ (b) $\Delta=2$, $n^{A} / n_{\mathrm{I}}=1.646+0.004 m(m=0, \ldots, 10)$. See the caption of Fig. 5.

$$
\hat{\mathcal{N}}_{i}^{B}=\frac{l_{b}}{l_{r}} \hat{n}^{A} \hat{T}^{1 / 2} G_{s}\left(M_{n}, M_{t}, \hat{T}, \frac{l_{r}}{\hat{n}^{A} l_{b}} \tilde{\Gamma}\right) a_{i},
$$

where

$$
a_{i}=\left\{\begin{array}{l}
t_{i} \quad\left(\text { for } \hat{v}_{j} t_{j} \geqslant 0\right), \\
-t_{i} \quad\left(\text { for } \hat{v}_{j} t_{j}<0\right) .
\end{array}\right.
$$

[Here, $a_{i}$ for $\hat{v}_{j} t_{j}=0$ is defined as $t_{i}$; see the sentence following that containing Eq. (12).] Note that the ratio $l_{r} / l_{b}$ appearing in the functions $\mathcal{F}_{s}$ and $G_{s}$ is given by $l_{r} / l_{b}=\hat{n}^{A} / \hat{T}^{1 / 2}$ for the GSB model. In order to complete the boundary condition, we need the relation between $\Delta$ and $\widetilde{\Gamma}$. This relation takes the following form:

$$
\Delta=\frac{\sqrt{\pi}}{4} \int \tilde{\Gamma} d s,
$$

where $d s$ is the line element along the sinusoidal boundary in the dimensionless $x_{1} x_{2}$ plane, and the range of integration is from $x_{2}=0$ to 2 . This relation is obtained by noting that $(\sqrt{\pi} / 2) n_{\mathrm{I}} l_{r} \widetilde{\Gamma}$ is the total number of the noncondensable gas in the Knudsen layer per unit area of the boundary [see the paragraph containing Eq. (15) in Sec. II C] and that its total number in the entire domain per unit width in $X_{3}$, i.e., $n_{a v}^{B}\left(2 L^{2}\right)$, is therefore given by $(\sqrt{\pi} / 2) n_{\mathrm{I}} l_{r} L \int \widetilde{\Gamma} d s$. Finally, these boundary conditions are supplemented by the condition $h\left(x_{2}=2\right)=h\left(x_{2}=0\right) \quad\left(h=\hat{\rho}^{A}, \hat{p}^{A}, \hat{v}_{i}\right.$, or $\left.\hat{T}\right)$ and $\tilde{\Gamma}\left(x_{2}=2\right)$

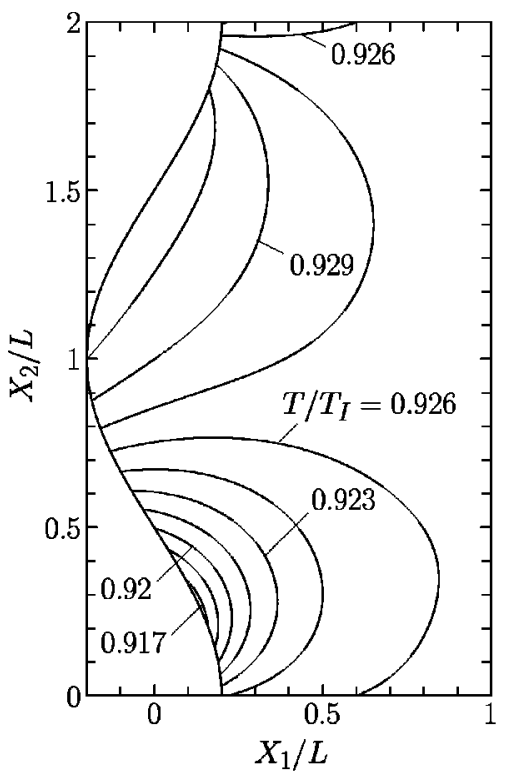

(a) pure vapor $(\Delta=0)$

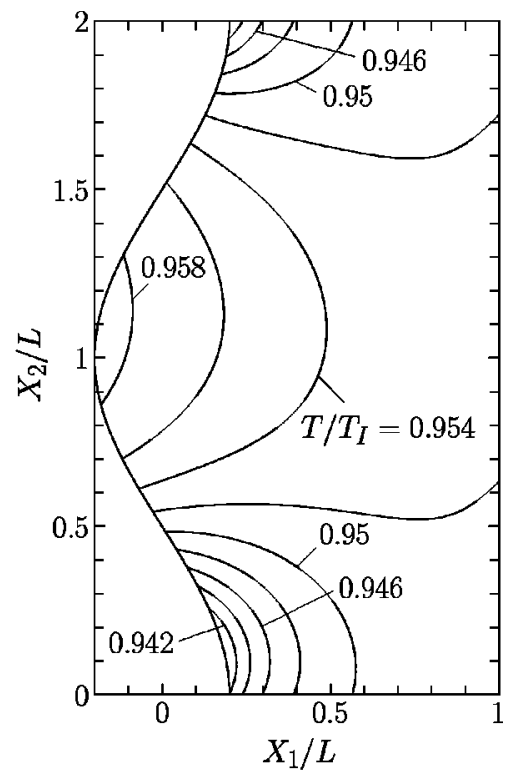

(b) $\Delta=2$
FIG. 9. Isolines of the temperature $T$ for $\hat{U}_{\mathrm{II}}=0.1$, $T_{\mathrm{II}} / T_{\mathrm{I}}=1, p_{\mathrm{II}} / p_{\mathrm{I}}=2$, and $A / L=0.2$. (a) Pure vapor $(\Delta$ $=0), \quad T / T_{\mathrm{I}}=0.917+0.0015 m(m=0, \ldots, 9)$; (b) $\Delta=2$, $T / T_{\mathrm{I}}=0.942+0.002 m(m=0, \ldots, 8)$. See the caption of Fig. 5. 
$=\widetilde{\Gamma}\left(x_{2}=0\right)$, which corresponds to the periodic boundary condition.

We solve this boundary-value problem numerically by means of a standard finite-difference method. Here, we omit the description about the numerical method and show only the results. The computation is carried out for $A / L=0.2$, $T_{\mathrm{II}} / T_{\mathrm{I}}=1$, and $p_{\mathrm{II}} / p_{\mathrm{I}}=2$.

Figure 5 shows the streamlines of the vapor flow for $\hat{U}_{\mathrm{II}}=0,0.1,0.2$, and 0.3 in the case of $\Delta=2$, and Fig. 6 shows those for the same $\hat{U}_{\mathrm{II}}$ in the case of $\Delta=4$. In these figures, the corresponding streamlines for a pure-vapor flow $(\Delta=0)$ are also shown by dashed lines. As is seen from the figures, the flow pattern of the vapor strongly depends on $\Delta$. (In the case of $\hat{U}_{\mathrm{II}}=0$, the pattern of the streamlines for $\Delta=2$ and that for $\Delta=4$ are quite similar [see Figs. 5(a) and 6(a)]. But, as is discussed in Ref. 18, the values of the quantities such as the flow speed $\left|v_{i}\right|$ and the number density $n^{A}$ are different (see Sec. 4 of Ref. 18).) Let us consider the case where $\hat{U}_{\text {II }}$ is finite [cf. Figs. 5(b)-5(d) and 6(b)-6(d)]. The streamlines of the vapor flow near the plane condensed phase show a similar tendency to those in the two-surface problem (Sec. III A). That is, the presence of the noncondensable gas in the Knudsen layer on the sinusoidal condensed phase weakens condensation there and thus evaporation on the plane condensed phase, so that the streamlines become more tangential to the plane condensed phase for larger values of $\Delta$. In the purevapor case $(\Delta=0)$, the shape of the condensing surface has a weak effect on the overall vapor flow, and its streamlines are nearly parallel and do not differ much from those of the two-surface problem. When the noncondensable gas is contained in the Knudsen layer on the condensing surface, the magnitude of the component of the flow velocity of the vapor normal to the condensed phase is reduced there. On the other hand, the magnitude of its tangential component is not reduced much because the noncondensable gas is mobile along the condensed phase and therefore does not give strong resistance. Therefore, the incidence of the streamlines becomes more tangential for larger values of $\Delta$. In this way, the vapor flow is more affected by the geometry of the condensing surface when $\Delta$ is large. When $\hat{U}_{\text {II }}=0$ [Figs. 5 (a) and 6(a)], the problem is symmetric with respect to the $X_{1}$ axis
TABLE IV. Mass-flow rate $\hat{\mathcal{M}}$ for various values of $\hat{U}_{\text {II }}$ and $\Delta$ in the case $T_{\mathrm{II}} / T_{\mathrm{I}}=1, p_{\mathrm{II}} / p_{\mathrm{I}}=2$, and $A / L=0.2$. Here, the molecules of the noncondensable gas are assumed to be mechanically identical with those of the vapor.

\begin{tabular}{lccc}
\hline \hline$\hat{U}_{\mathrm{II}} \backslash \Delta$ & 0 & 2 & 4 \\
\hline 0 & 0.4987 & 0.3734 & 0.2863 \\
0.1 & 0.4984 & 0.3580 & 0.2773 \\
0.2 & 0.4979 & 0.3442 & 0.2727 \\
0.3 & 0.4989 & 0.3435 & 0.2593 \\
\hline \hline
\end{tabular}

and thus $\hat{\mathcal{N}}_{i}^{B} t_{i}=0$ holds identically. Therefore, it follows from Eqs. (13) and (32) that $M_{t}=0$ on the sinusoidal condensed phase. In other words, the vapor condenses perpendicularly there. In contrast, the vapor condenses obliquely in the purevapor case. This difference has been pointed out in Ref. 18 .

The isolines of the flow speed $\left|v_{i}\right|$, those of the number density $n^{A}$, and those of the temperature $T$ are shown in Figs. 7-9, respectively, for $\hat{U}_{\text {II }}=0.1$. In Figs. 7-9, the isolines of the quantities for the pure vapor $(\Delta=0)$ are shown in (a), while those for $\Delta=2$ in (b). Since the motion of the plane condensed phase is relatively slow $\left(\hat{U}_{\mathrm{II}}=0.1\right)$, the density and temperature variations are small. Therefore, a small error can change isolines significantly. It should be understood that the isolines in Figs. 8 and 9 give information about the almost uniform values of the number density and temperature of the vapor but do not show the detailed structure of their small variations accurately. Let $\mathcal{M}$ be the rate of the mass of the vapor evaporating from the part $0 \leqslant X_{1} \leqslant 2 L$ of the plane condensed phase per unit time and per unit width in the $X_{3}$ direction. Its dimensionless counterpart $\hat{\mathcal{M}}$, defined by $\hat{\mathcal{M}}$ $=\mathcal{M} / m^{A} n_{\mathrm{I}}\left(2 k T_{\mathrm{I}} / m^{A}\right)^{1 / 2} L$, is shown in Table IV.

In summary of all the results presented here, the noncondensable gas with an infinitesimal average concentration completely changes the global two-dimensional flow field of the vapor.

The quantity $\widetilde{\Gamma}$, which is a function of the position on the boundary, is a measure of accumulation of the noncondensable gas inside the thickless Knudsen layer on the condensing boundary. The distribution of $\widetilde{\Gamma}$ along the sinusoidal con-

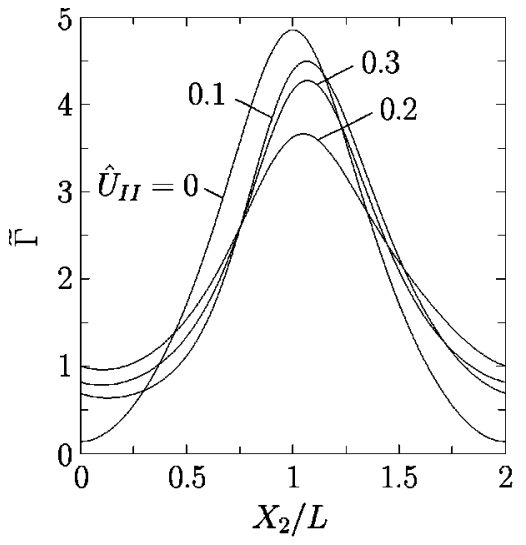

(a) $\Delta=2$

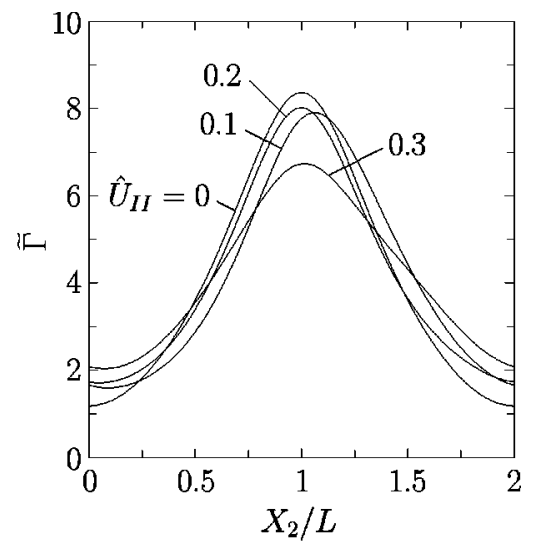

(b) $\Delta=4$
FIG. 10. Distribution of $\widetilde{\Gamma}$ along the sinusoidal condensed phase for various values of $\hat{U}_{\mathrm{II}}$ in the case of $T_{\mathrm{II}} / T_{\mathrm{I}}$ $=1, p_{\mathrm{II}} / p_{\mathrm{I}}=2$, and $A / L=0.2$. (a) $\Delta=2$, (b) $\Delta=4$. See the caption of Fig. 5 . 


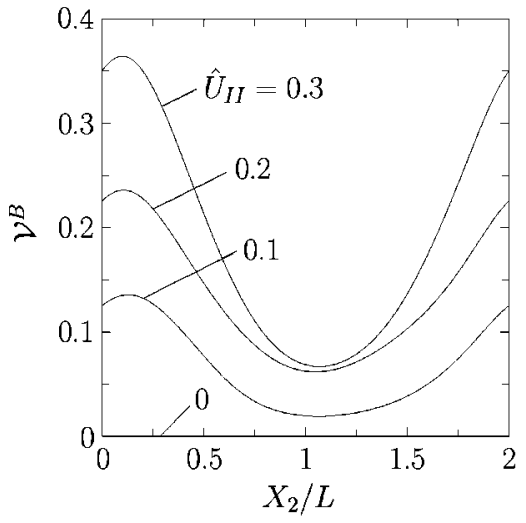

(a) $\Delta=2$

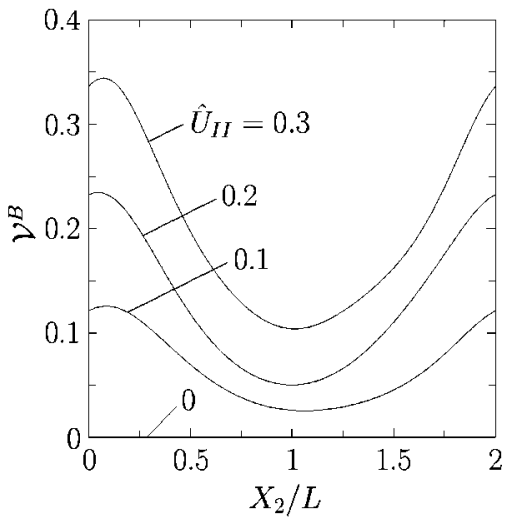

(b) $\Delta=4$
FIG. 11. Distribution of $\mathcal{V}^{B}$ along the sinusoidal condensed phase for various values of $\hat{U}_{\mathrm{II}}$ in the case of $T_{\mathrm{II}} / T_{\mathrm{I}}=1, p_{\mathrm{II}} / p_{\mathrm{I}}=2$, and $A / L=0.2$. (a) $\Delta=2$, (b) $\Delta=4$. Here, $\mathcal{V}^{B}=0$ holds identically for $\hat{U}_{\mathrm{II}}=0$. See the caption of Fig. 5 . densed phase is shown in Fig. 10 for various values of $\hat{U}_{\mathrm{II}}$ in the case of $\Delta=2$ [Fig. 10(a)] and 4 [Fig. 10(b)]. Let us define a kind of average flow speed $\mathcal{V}^{B}$ of the noncondensable gas inside the Knudsen layer along the sinusoidal condensed phase by

$$
\tilde{\Gamma} \mathcal{V}^{B}=\hat{\mathcal{N}}_{i}^{B} t_{i}
$$

Recall that $\hat{\mathcal{N}}_{i}^{B} t_{i}$, which is the total particle flux of the noncondensable gas in the Knudsen layer, is constant along the boundary [Eq. (14)]. The distribution of $\mathcal{V}^{B}$ along the sinusoidal condensed phase is shown in Fig. 11 for $\hat{U}_{\mathrm{II}}=0,0.1$, 0.2 , and 0.3 in the case of $\Delta=2$ [Fig. 11(a)] and 4 [Fig. 11(b)]. When $\hat{U}_{\mathrm{II}}=0, \mathcal{V}^{B}$ vanishes identically (see Sec. 4 of Ref. 18). The average speed $\mathcal{V}^{B}$ naturally increases with the increase of $\hat{U}_{\mathrm{II}}$.

Finally, we show some results for relatively small $\Delta$. If the amount of the noncondensable gas is large enough, it distributes over the entire boundary (see Fig. 10). On the other hand, when the amount of the noncondensable gas becomes small, it may vanish (or equivalently, $\widetilde{\Gamma}$ may vanish) in a certain part of the boundary. Since we are considering a steady flow, we need to assume that the part which contains the noncondensable gas does not move. This means that its particle flux $\hat{\mathcal{N}}_{i}^{B} t_{i}$ vanishes and thus $M_{t}=0$ holds because of Eq. (13) with nonzero $\widetilde{\Gamma}$. To summarize, Eq. (30) with $M_{t}$ $=0$ holds in the part which contains the noncondensable gas, and Eq. (30) with $\widetilde{\Gamma}=0$ holds in the part which does not contain it. Figure 12 shows the distributions of $\widetilde{\Gamma}$ and $\mathcal{V}^{B}$ along the sinusoidal boundary, in the case of $\hat{U}_{\mathrm{II}}=0.1$, for several values of $\Delta$, i.e., $\Delta=2,1,0.5,0.1$, and 0.05 . When $\Delta$ is not small, the noncondensable gas distributes over the whole boundary. But for small $\Delta(\Delta=0.1,0.05)$, the noncondensable gas tends to disappear from the part $0 \leqslant X_{2} / L \leqq 1$ and from the part near $X_{2} / L=2$. (The $\mathcal{V}^{B}$ does not have a meaning in the part without the noncondensable gas.) At the left-side edge of the part with the noncondensable gas in Fig. 12(a), the slope of the distribution of $\widetilde{\Gamma}$ tends to become very steep. If the distribution of a physical quantity, e.g., $\hat{\rho}^{A}, \hat{v}_{i}$, or $\hat{T}$, along the sinusoidal condensed phase is plotted as a function of $X_{2} / L$, the derivative of the curve seems to be discontinuous at the corresponding point, and its limit from the right seems to be (plus or minus) infinity. In contrast, at the right-hand edge of the part with the noncondensable gas, $\tilde{\Gamma}$ disappears smoothly and no singularity appears, that is, the curve of any physical quantity is smooth at the corresponding point. Here, we should recall that the Euler set of equations is derived from the Hilbert solution of the Boltzmann equation with a moderately varying property. ${ }^{18}$ Therefore, if such a singularity as described above appears in the physical quantities, it violates the assumption for the Hilbert solution. In other words, the present fluid-dynamic system is not theoretically applicable to such situations. In order to treat this type of flow correctly, we need a two-dimensional analysis based on kinetic theory in the neighborhood of the singular point.
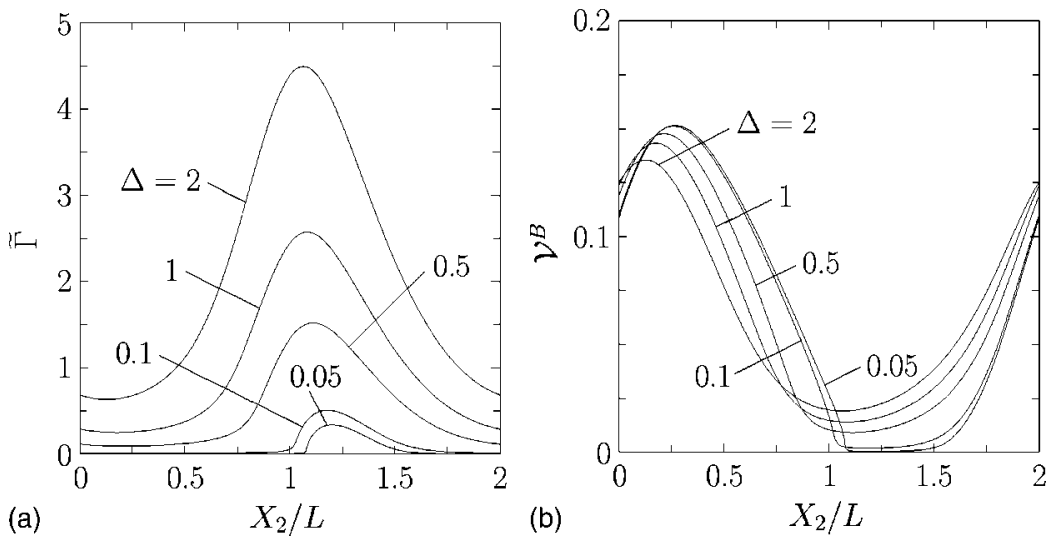

FIG. 12. Distribution of $\widetilde{\Gamma}$ (a) and that of $\mathcal{V}^{B}$ (b) along the sinusoidal condensed phase for various values of $\Delta$ for $\hat{U}_{\mathrm{II}}=0.1, T_{\mathrm{II}} / T_{\mathrm{I}}=1, p_{\mathrm{II}} / p_{\mathrm{I}}=2$, and $A / L=0.2$. See the caption of Fig. 5. 


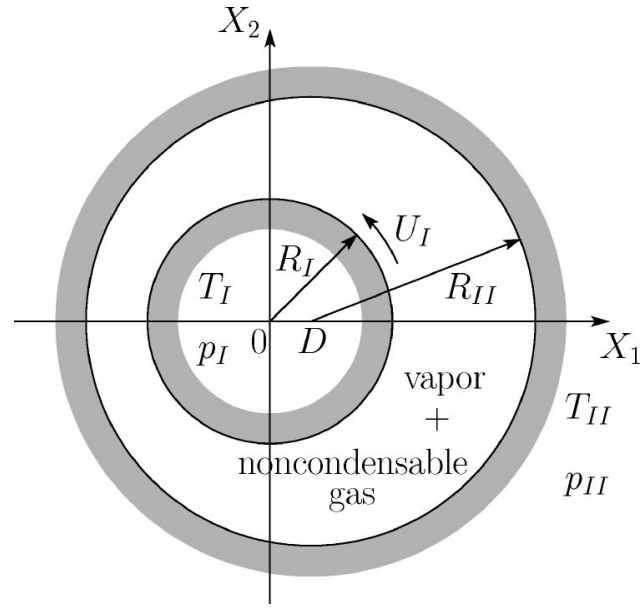

FIG. 13. Vapor flow between cylindrical condensed phases in the presence of a noncondensable gas.

\section{Vapor flows between two noncoaxial cylindrical condensed phases}

In this section, we consider a vapor ( $A$ component $)$ and a noncondensable gas ( $B$ component) between two noncoaxial circular cylinders made of the condensed phase as shown in Fig. 13, where the $X_{1}, X_{2}$, and $X_{3}$ axes are assumed to form the right-hand system. Let $R_{\mathrm{I}}$ (or $R_{\mathrm{II}}$ ) be the radius of the inner (or outer) cylinder, $T_{\mathrm{I}}$ (or $T_{\mathrm{II}}$ ) the temperature of the inner (or outer) cylinder, $D$ the distance between the two axes, and $p_{\text {I }}$ (or $\left.p_{\mathrm{II}}\right)$ the saturation pressure of the vapor at temperature $T_{\mathrm{I}}$ ( or $T_{\mathrm{II}}$ ). The inner cylinder may be rotating (counterclockwise in Fig. 13) around its axis with a constant angular velocity, whereas the outer cylinder is kept at rest. We denote by $U_{\mathrm{I}}$ the speed of the surface of the inner cylinder and take $R_{\mathrm{I}}$ as the reference length and $T_{\mathrm{I}}, p_{\mathrm{I}}$, and $n_{\mathrm{I}}$ $=p_{\mathrm{I}} / k T_{\mathrm{I}}$ as the reference quantities, i.e., $L=R_{\mathrm{I}}, T_{r}=T_{\mathrm{I}}, p_{r}$ $=p_{\mathrm{I}}$, and $n_{r}=n_{\mathrm{I}}$. Then the Knudsen number of the system is given by $\mathrm{Kn}=l_{r} / R_{\mathrm{I}}$ with $l_{r}$ being the mean free path of the vapor molecules in the equilibrium state at rest with temperature $T_{\mathrm{I}}$ and pressure $p_{\mathrm{I}}$ (or molecular number density $n_{\mathrm{I}}$ ). As before, the average molecular number density of the noncondensable gas over the whole domain is denoted by $n_{a v}^{B}$ and is put in the form $n_{a v}^{B} / n_{\mathrm{I}}=\Delta \mathrm{Kn}$, where $\Delta$ is a constant to be specified. The problem is characterized by the following parameters:

$$
\frac{T_{\mathrm{II}}}{T_{\mathrm{I}}}, \quad \frac{p_{\mathrm{II}}}{p_{\mathrm{I}}}, \quad \hat{U}_{\mathrm{I}}=\frac{U_{\mathrm{I}}}{\left(2 k T_{\mathrm{I}} / m^{A}\right)^{1 / 2}}, \quad \frac{R_{\mathrm{II}}}{R_{\mathrm{I}}}, \quad \frac{D}{R_{\mathrm{I}}}, \quad \mathrm{Kn}, \quad \Delta .
$$

We investigate the steady behavior of the vapor and the effect of the trace of the noncondensable gas in the continuum limit $(\mathrm{Kn} \rightarrow 0)$.

The behavior of the vapor is described by the Euler equations (5a)-(5d). Let us consider the case where the vapor is evaporating from the inner cylinder and condensing onto the outer cylinder and assume that the speed of condensation on the outer cylinder is subsonic. Then the boundary condition on the inner cylinder $\left(x_{1}^{2}+x_{2}^{2}=1\right)$, where evaporation is taking place, is given by

$$
\begin{aligned}
& M_{n} \leqslant 1, \quad \hat{v}_{j} t_{j}=\hat{U}_{\mathrm{I}}, \\
& \hat{p}^{A}=h_{1}\left(M_{n}\right), \quad \hat{T}=h_{2}\left(M_{n}\right),
\end{aligned}
$$

where

$$
M_{n}=\sqrt{6 / 5} \hat{T}^{-1 / 2}\left|\hat{v}_{j} n_{j}\right|,
$$

and $n_{i}$ and $t_{i}$ are the unit normal and tangential vectors to the inner cylinder, $n_{i}$ pointing into the region of the vapor, and $n_{i}, t_{i}$, and the $X_{3}$ axis forming the right-hand system. In Eqs. (37) and (38) and Eqs. (39)-(42) below, the notation $(\cdot)_{b}$ is omitted as in Sec. III B. On the other hand, the boundary condition on the outer cylinder $\left[\left(x_{1}-D / R_{\mathrm{I}}\right)^{2}+x_{2}^{2}=\left(R_{\mathrm{II}} / R_{\mathrm{I}}\right)^{2}\right]$, where condensation is taking place, is given by

$$
\hat{p}^{A}=\frac{p_{\mathrm{II}}}{p_{\mathrm{I}}} \mathcal{F}_{s}\left(M_{n}, M_{t}, \frac{T_{\mathrm{I}}}{T_{\mathrm{II}}} \hat{T}, \frac{l_{r}}{\hat{n}^{A} l_{b}} \tilde{\Gamma}\right),
$$

where

$$
\begin{aligned}
& M_{n}=\sqrt{6 / 5} \hat{T}^{-1 / 2}\left|\hat{v}_{j} n_{j}\right|, \\
& M_{t}=\sqrt{6 / 5} \hat{T}^{-1 / 2}\left|\hat{v}_{j} t_{j}\right|,
\end{aligned}
$$

and $n_{i}$ and $t_{i}$ are the unit normal and tangential vectors to the outer cylinder, $n_{i}$ pointing into the region of the vapor, and $n_{i}, t_{i}$, and the $X_{3}$ axis forming the right-hand system. In addition, Eq. (14) holds along the outer cylinder, where $\hat{\mathcal{N}}_{i}^{B}$ is given by

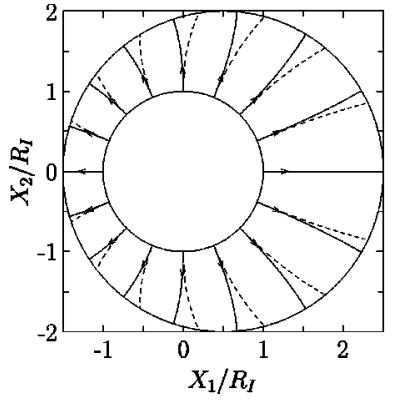

(a) $\hat{U}_{I}=0$

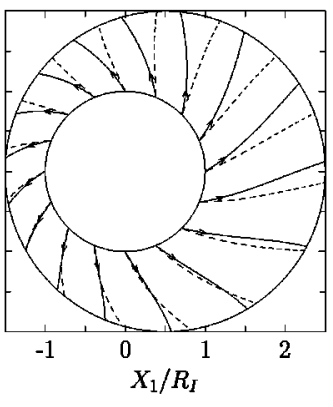

(b) $\hat{U}_{I}=0.1$

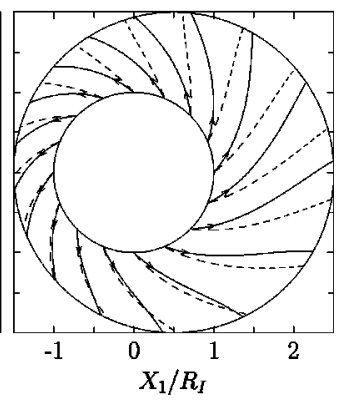

(c) $\hat{U}_{I}=0.2$
FIG. 14. Streamlines of the vapor flow for $\Delta=2$ in the case of $T_{\mathrm{II}} / T_{\mathrm{I}}=1, p_{\mathrm{II}} / p_{\mathrm{I}}=0.5, R_{\mathrm{II}} / R_{\mathrm{I}}=2$, and $D / R_{\mathrm{I}}=0.5$. (a) $\hat{U}_{\mathrm{I}}=0$, (b) $\hat{U}_{\mathrm{I}}=0.1$, (c) $\hat{U}_{\mathrm{I}}=0.2$. The corresponding streamlines in the pure-vapor case $(\Delta=0)$ are shown by dashed lines. Here, the molecules of the noncondensable gas are assumed to be mechanically identical with those of the vapor. 


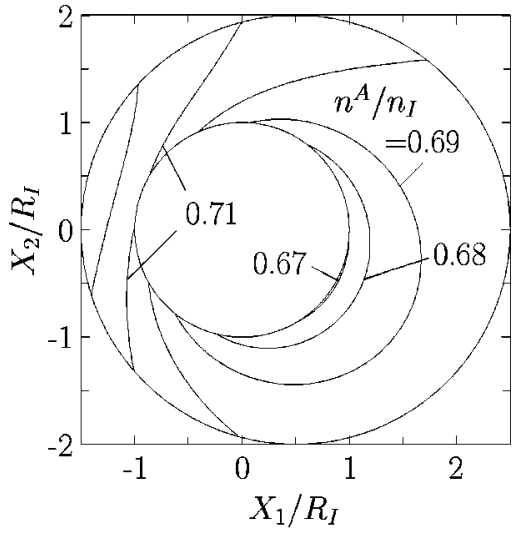

(a) $\Delta=0$

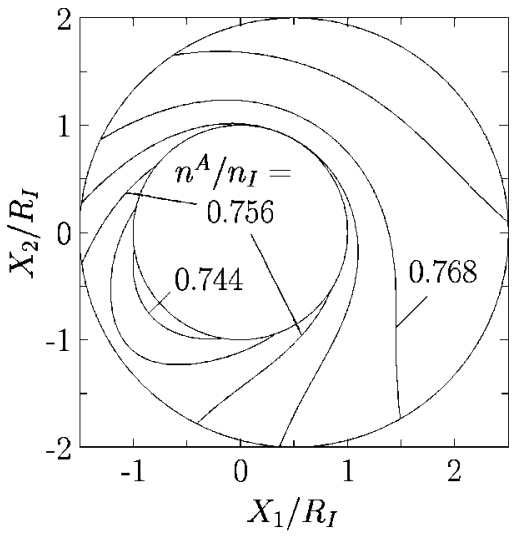

(b) $\Delta=2$
FIG. 15. Isolines of the number density $n^{A}$ for $\hat{U}_{\mathrm{I}}=0.1$ in the case $T_{\mathrm{II}} / T_{\mathrm{I}}=1, p_{\mathrm{II}} / p_{\mathrm{I}}=0.5, R_{\mathrm{II}} / R_{\mathrm{I}}=2$, and $D / R_{\mathrm{I}}$ $=0.5$. (a) Pure vapor $(\Delta=0), n^{A} / n_{\mathrm{I}}=0.67+0.01 \mathrm{~m}(\mathrm{~m}$ $=0, \ldots, 5) ; \quad$ (b) $\quad \Delta=2, \quad n^{A} / n_{\mathrm{I}}=0.744+0.006 m(m$ $=0, \ldots, 5)$. See the caption of Fig. 14 .

$$
\hat{\mathcal{N}}_{i}^{B}=\frac{l_{b}}{l_{r}} \hat{n}^{A} \hat{T}^{1 / 2} G_{s}\left(M_{n}, M_{t}, \frac{T_{\mathrm{I}}}{T_{\mathrm{II}}} \hat{T}, \frac{l_{r}}{\hat{n}^{A} l_{b}} \tilde{\Gamma}\right) a_{i},
$$

with

$$
a_{i}=\left\{\begin{array}{ccc}
t_{i} & \text { for } & \hat{v}_{j} t_{j} \geqslant 0, \\
-t_{i} & \text { for } & \hat{v}_{j} t_{j}<0 .
\end{array}\right.
$$

[Here, $a_{i}$ for $\hat{v}_{j} t_{j}=0$ is defined as $t_{i}$; see the sentence following that containing Eq. (12).] Finally, the relation between $\Delta$ and $\widetilde{\Gamma}$ is given by

$$
\Delta=\frac{1}{2 \sqrt{\pi}}\left[\left(\frac{R_{\mathrm{II}}}{R_{\mathrm{I}}}\right)^{2}-1\right]^{-1} \int \tilde{\Gamma} d s,
$$

where $d s$ is the (dimensionless) line element along the outer cylinder, and the integration is carried out over the whole circle. This relation is obtained in a similar way as Eq. (34).

We analyze the above boundary-value problem of the Euler set of equations by a finite-difference method. The result for $T_{\mathrm{II}} / T_{\mathrm{I}}=1, p_{\mathrm{II}} / p_{\mathrm{I}}=0.5, R_{\mathrm{II}} / R_{\mathrm{I}}=2$, and $D / R_{\mathrm{I}}=0.5$ is shown in Figs. 14-17. Figure 14 shows the streamlines of the vapor flow for $\hat{U}_{\mathrm{I}}=0,0.1$, and 0.2 in the case of $\Delta=2$. In the figure, the streamlines for the corresponding pure-vapor case $(\Delta=0)$ are also shown by dashed lines. Figure 15 shows the isolines of the molecular number density of the vapor $n^{A}$ in the case of $\hat{U}_{\mathrm{I}}=0.1$, where the isolines for the pure-vapor case $(\Delta=0)$ are shown in (a) and those for $\Delta=2$ in (b). Figure 16 shows the distribution of $\widetilde{\Gamma}$ and that of $\mathcal{V}^{B}$ along the outer cylinder for $\Delta=2$. Here, $\mathcal{V}^{B}$ is the same as that used in Sec. III B [Eq. (35)], and $\theta(0 \leqslant \theta<2 \pi)$ is the angle between the $X_{1}$ axis and the line connecting a point on the outer cylinder and its axis [the point $(D, 0)$ in Fig. 13] measured counterclockwise. Let $\mathcal{M}=m^{A} n_{\mathrm{I}}\left(2 k T_{\mathrm{I}} / m^{A}\right)^{1 / 2} R_{\mathrm{I}} \hat{\mathcal{M}}$ be the total mass-flow rate of the vapor evaporating from the inner cylinder (per unit width in the $X_{3}$ direction and per unit time). The dimensionless mass-flow rate $\hat{\mathcal{M}}$ versus $\Delta$ for $\hat{U}_{\mathrm{I}}=0,0.1,0.2$, and 0.3 is shown in Fig. 17 .

The features of the streamlines can basically be understood from the discussion in Sec. III B. The dimensionless mass-flow rate $\hat{\mathcal{M}}$ naturally decreases with the increase of $\Delta$. Faster rotation of the inner cylinder leads to a pressure rise on the outer cylinder because of stronger centrifugal effect and thus causes stronger condensation. Therefore, $\hat{\mathcal{M}}$ increases as $\hat{U}_{\mathrm{I}}$ increases. The results shown here again demonstrate that the noncondensable gas with an infinitesimal average concentration has a significant effect on the overall vapor flow field.

\section{CONCLUDING REMARKS}

In a previous paper, ${ }^{18}$ we derived the fluid-dynamic system that describes steady flows of a vapor around its condensed phase, on the surface of which evaporation or condensation is taking place, in the continuum limit $(\mathrm{Kn} \rightarrow 0)$ in the presence of a noncondensable gas whose amount is in-

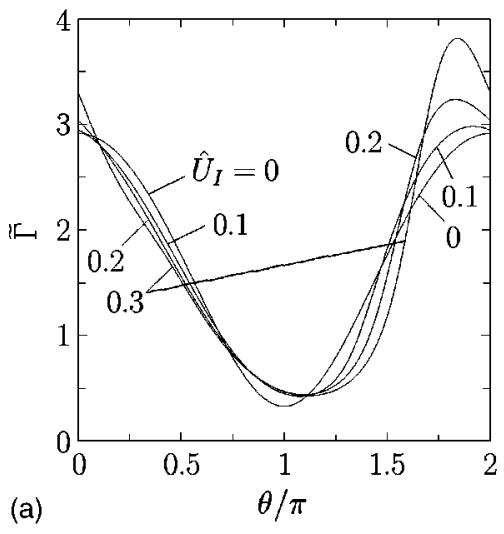

(a)

Downloaded 04 Jun 2007 to 130.54.110.22. Redistribution subject to AIP license or copyright, see http://pof.aip.org/pof/copyright.jsp
FIG. 16. Distribution of $\widetilde{\Gamma}$ and that of $\mathcal{V}^{B}$ along the outer cylinder for various $\hat{U}_{\text {I }}$ in the case $\Delta=2$. (a) $\widetilde{\Gamma}$, (b) $\mathcal{V}^{B}$. Here, $\theta(0 \leqslant \theta<2 \pi)$ is the angle between the $X_{1}$ axis and the line connecting a point on the outer cylinder and its axis [the point $(D, 0)$ in Fig. 13] measured counterclockwise. In (b), $\mathcal{V}^{B}=0$ holds identically in the case $\hat{U}_{\mathrm{I}}=0$. See the caption of Fig. 14 . (b)

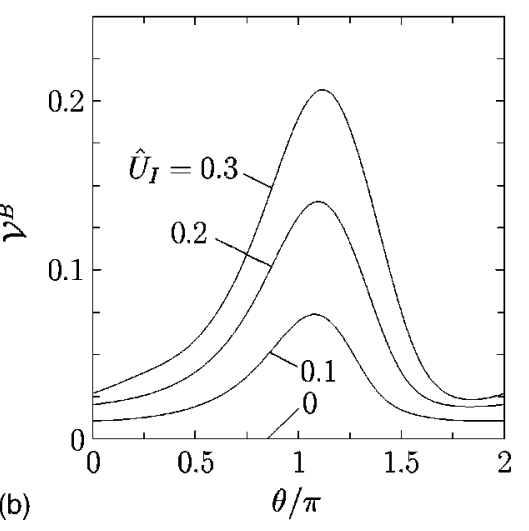




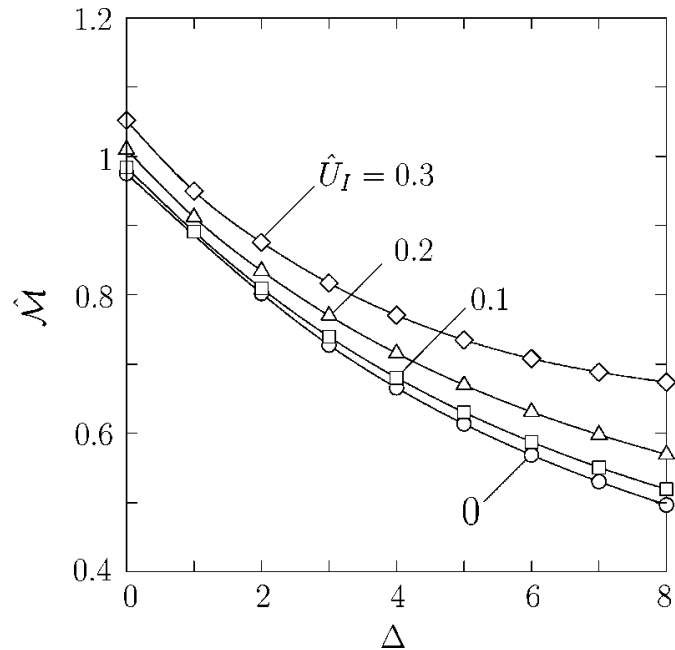

FIG. 17. $\hat{\mathcal{M}}$ vs $\Delta$ for various $\hat{U}_{\mathrm{I}}$. The symbols $\bigcirc, \square, \triangle$, and $\diamond$ indicate the numerical data, which are connected by spline curves. See the caption of Fig. 14.

finitesimal in comparison with that of the vapor. In the case of a closed domain, the amount of the noncondensable gas is expressed more precisely in such a way that its average concentration is infinitesimal $[O(\mathrm{Kn})]$. In the same paper, we pointed out that such a small amount of the noncondensable gas can affect the overall vapor flow significantly, accumulating in the Knudsen layer with an infinitesimal thickness on the condensing surface. The present paper is devoted to the application of the fluid-dynamic system mentioned above to some concrete problems that demonstrate the effect of the trace of the noncondensable gas clearly.

The fluid-dynamic system consists of the set of the compressible Euler equations and its boundary conditions constructed numerically under the following assumptions: (i) the mechanical property of the molecules of the vapor and that of the noncondensable gas are the same and (ii) the model Boltzmann equations (the BGK and GSB models) describe the behavior in the Knudsen layers (Sec. II). On the basis of this system, the two-surface problem of evaporation and condensation (Sec. III A), vapor flows between a plane and a sinusoidal condensed phase (Sec. III B), and vapor flows between two noncoaxial cylindrical condensed phases (Sec. III C) were investigated numerically. In these problems, it is clearly seen that the noncondensable gas with an infinitesimal average concentration has significant effects on the overall vapor flows in the continuum limit.

\section{ACKNOWLEDGMENTS}

The present work was supported by the Grant-in-Aid for Scientific Research (Grant No. 14350047) from the Japan Society for the Promotion of Science and by the Center of Excellence for Research and Education on Complex Functional Mechanical Systems (the COE program of the Ministry of Education, Culture, Sports, Science and Technology, Japan).

${ }^{1}$ M. N. Kogan, "Kinetic theory in aerothermodynamics," Prog. Aerosp. Sci. 29, 271 (1992).
${ }^{2}$ T. Ytrehus, "Molecular-flow effects in evaporation and condensation at interfaces," Multiphase Sci. Technol. 9, 205 (1997).

${ }^{3}$ C. Cercignani, Rarefied Gas Dynamics: From Basic Concepts to Actual Calculations (Cambridge University Press, Cambridge, 2000).

${ }^{4}$ A. K. Rebrov, "Nonequilibrium processes at evaporating and condensing surfaces," in Rarefied Gas Dynamics, edited by T. J. Bartel and M. A. Gallis (AIP, Melville, 2001), p. 557.

${ }^{5}$ Y. Sone, Kinetic Theory and Fluid Dynamics, Modeling and Simulation in Science, Engineering and Technology (Birkhäuser, Boston, 2002).

${ }^{6}$ Y. Sone, Molecular Gas Dynamics, Modeling and Simulation in Science, Engineering and Technology (Birkhäuser, Boston, 2005).

${ }^{7}$ Y. Sone and Y. Onishi, "Kinetic theory of evaporation and condensationHydrodynamic equation and slip boundary condition-," J. Phys. Soc. Jpn. 44, 1981 (1978).

${ }^{8}$ Y. Onishi and Y. Sone, "Kinetic theory of slightly strong evaporation and condensation-Hydrodynamic equation and slip boundary condition for finite Reynolds number-," J. Phys. Soc. Jpn. 47, 1676 (1979).

${ }^{9}$ Y. Sone, "Asymptotic theory of a steady flow of a rarefied gas past bodies for small Knudsen numbers," in Advances in Kinetic Theory and Continuum Mechanics, edited by R. Gatignol and Soubbaramayer (Springer, Berlin, 1991), p. 19.

${ }^{10} \mathrm{~K}$. Aoki and Y. Sone, "Gas flows around the condensed phase with strong evaporation or condensation-Fluid dynamic equation and its boundary condition on the interface and their application-," in Advances in Kinetic Theory and Continuum Mechanics, edited by R. Gatignol and Soubbaramayer (Springer, Berlin, 1991), p. 43.

${ }^{11}$ Y. Onishi, "Kinetic theory based nonlinear treatment for motions of a binary gas mixture involving slightly strong evaporation and condensation processes-A system of macroscopic equations, the boundary conditions and the Knudsen-layer corrections," ZAMP 43, 875 (1992).

${ }^{12} \mathrm{Y}$. Onishi, "Kinetic theory based nonlinear treatment for motions of a binary gas mixture involving slightly strong evaporation and condensation processes II-A system of macroscopic equations, the boundary conditions and the Knudsen-layer corrections," ZAMP 44, 828 (1993).

${ }^{13} \mathrm{~K}$. Aoki, S. Takata, and S. Kosuge, "Vapor flows caused by evaporation and condensation on two parallel plane surfaces: Effect of the presence of a noncondensable gas," Phys. Fluids 10, 1519 (1998).

${ }^{14}$ S. Takata, K. Aoki, and T. Muraki, "Behavior of a vapor-gas mixture between two parallel plane condensed phases in the continuum limit," in Rarefied Gas Dynamics, edited by R. Brun, R. Campargue, R. Gatignol, and J.-C. Lengrand (Cépaduès-Éditions, Toulouse, 1999), Vol. 1, p. 479.

${ }^{15} \mathrm{~S}$. Takata and K. Aoki, "Two-surface problems of a multicomponent mixture of vapors and noncondensable gases in the continuum limit in the light of kinetic theory," Phys. Fluids 11, 2743 (1999).

${ }^{16} \mathrm{~S}$. Takata and K. Aoki, "The ghost effect in the continuum limit for a vapor-gas mixture around condensed phases: Asymptotic analysis of the Boltzmann equation," Transp. Theory Stat. Phys. 31, 205 (2001); 30, 289(E) (2002).

${ }^{17} \mathrm{~K}$. Aoki, "The behavior of a vapor-gas mixture in the continuum limit: Asymptotic analysis based on the Boltzmann equation," in Rarefied Gas Dynamics, edited by T. J. Bartel and M. A. Gallis (AIP, Melville, 2001), p. 565.

${ }^{18}$ K. Aoki, S. Takata, and S. Taguchi, "Vapor flows with evaporation and condensation in the continuum limit: Effect of a trace of noncondensable gas," Eur. J. Mech. B/Fluids 22, 51 (2003).

${ }^{19} \mathrm{~S}$. Taguchi, K. Aoki, and S. Takata, "Vapor flows condensing at incidence onto a plane condensed phase in the presence of a noncondensable gas. I. Subsonic condensation," Phys. Fluids 15, 689 (2003).

${ }^{20} \mathrm{~S}$. Taguchi, K. Aoki, and S. Takata, "Vapor flows condensing at incidence onto a plane condensed phase in the presence of a noncondensable gas. II. Supersonic condensation," Phys. Fluids 16, 79 (2004).

${ }^{21} \mathrm{~T}$. Ytrehus, "Theory and experiments on gas kinetics in evaporation," in Rarefied Gas Dynamics, edited by J. L. Potter (AIAA, New York, 1977), p. 1197.

${ }^{22}$ Y. Sone, "Kinetic theory of evaporation and condensation-Linear and nonlinear problems-," J. Phys. Soc. Jpn. 45, 315 (1978).

${ }^{23}$ Y. Sone and H. Sugimoto, "Strong evaporation from a plane condensed phase," in Adiabatic Waves in Liquid-Vapor Systems, edited by G. E. A. Meier and P. A. Thompson (Springer, Berlin, 1990), p. 293.

${ }^{24}$ Y. Sone, "Kinetic theoretical studies of the half-space problem of evaporation and condensation," Transp. Theory Stat. Phys. 29, 227 (2000).

${ }^{25}$ Y. Sone, K. Aoki, and T. Doi, "Kinetic theory analysis of gas flows condensing on a plane condensed phase: Case of a mixture of a vapor and a noncondensable gas," Transp. Theory Stat. Phys. 21, 297 (1992). 
${ }^{26} \mathrm{~K}$. Aoki and T. Doi, "High-speed vapor flows condensing on a plane condensed phase in the presence of a noncondensable gas," in Rarefied Gas Dynamics: Theory and Simulations, edited by B. D. Shizgal and D. P. Weaver (AIAA, Washington, DC, 1994), p. 521.

${ }^{27}$ P. L. Bhatnagar, E. P. Gross, and M. Krook, "A model for collision processes in gases. I. Small amplitude processes in charged and neutral onecomponent systems," Phys. Rev. 94, 511 (1954).

${ }^{28} \mathrm{P}$. Welander, "On the temperature jump in a rarefied gas," Ark. Fys. 7, 507 (1954).

${ }^{29}$ M. N. Kogan, "On the equations of motion of a rarefied gas," J. Appl. Math. Mech. 22, 597 (1958).

${ }^{30}$ Y. Sone and K. Aoki, Molecular Gas Dynamics (Asakura, Tokyo, 1994) (in Japanese).
${ }^{31}$ E. Kreyszig, Differential Geometry (Dover, New York, 1991), Chap. V.

${ }^{32}$ V. Garzó, A. Santos, and J. J. Brey, "A kinetic model for a multicomponent gas,” Phys. Fluids A 1, 380 (1989).

${ }^{33}$ See EPAPS Document No. E-PHFLE6-15-504303 for tables and figures. A direct link to this document may be found in the online article's HTML reference section. The document may also be reached via the EPAPS homepage (http://www.aip.org/pubservs/epaps.html) or from ftp.aip.org in the directory /epaps/. See the EPAPS homepage for more information.

${ }^{34}$ See EPAPS Document No. E-PHFLE6-16-503401 for tables and figures. See Ref. 33. 